

\title{
GNREL
}

\section{GeoRePORT Protocol Volume II: Geological Assessment Tool}

\author{
and Patrick F. Dobson ${ }^{2}$ \\ 1 National Renewable Energy Laboratory \\ 2 Lawrence Berkeley National Laboratory
}

Katherine R. Young, ${ }^{1}$ Anna Wall, ${ }^{1}$ Alex Badgett, ${ }^{1}$

\section{Suggested Citation}

Young, Katherine R., Anna Wall, Alex Badgett, and Patrick F. Dobson. 2019.

GeoRePORT Protocol Volume II: Geological Assessment Tool. Golden, CO: National Renewable Energy Laboratory. NREL/TP-4A00-73172.

https://www.nrel.gov/docs/fy19osti/73172.pdf.

NREL is a national laboratory of the U.S. Department of Energy Office of Energy Efficiency \& Renewable Energy Operated by the Alliance for Sustainable Energy, LLC

This report is available at no cost from the National Renewable Energy Laboratory (NREL) at www.nrel.gov/publications.

Contract No. DE-AC36-08GO28308
Technical Report NREL/TP-4A00-73172 September 2019

National Renewable Energy Laboratory 15013 Denver West Parkway Golden, CO 80401

303-275-3000 • www.nrel.gov 


\section{NOTICE}

This work was authored in part by the National Renewable Energy Laboratory, operated by Alliance for Sustainable Energy, LLC, for the U.S. Department of Energy (DOE) under Contract No. DE-AC36-08G028308. Funding provided by U.S. Department of Energy Office of Energy Efficiency and Renewable Energy Geothermal Technologies Office. The views expressed herein do not necessarily represent the views of the DOE or the U.S. Government.

This report is available at no cost from the National Renewable Energy Laboratory (NREL) at www.nrel.gov/publications.

U.S. Department of Energy (DOE) reports produced after 1991 and a growing number of pre-1991 documents are available free via www.OSTI.gov.

Cover Photos by Dennis Schroeder: (clockwise, left to right) NREL 51934, NREL 45897, NREL 42160, NREL 45891, NREL 48097, NREL 46526.

NREL prints on paper that contains recycled content. 


\section{ACKNOWLEDGMENTS}

This work was authored in part by the National Renewable Energy Laboratory, operated by Alliance for Sustainable Energy, LLC, for the U.S. Department of Energy (DOE) under Contract No. DE-AC3608GO28308. Funding provided by the U.S. Department of Energy Office of Energy Efficiency and Renewable Energy Geothermal Technologies Office. This work was also supported by the U.S. Department of Energy under Contract No. DE-AC02-05CH11231 with Lawrence Berkeley National Laboratory (LBNL). The views expressed herein do not necessarily represent the views of the DOE or the U.S. Government.

The authors wish to thank Colin Williams (U.S. Geological Survey); Paul Brophy (EGS, Inc.); Graeme Beardsmore (Hot Dry Rocks); Ann Robertson-Tait (GeothermEx); Ben Matek (Geothermal Energy Association); Mark Ziegenbein, Eric Hass, and Jay Nathwani (DOE); Brittany Segneri (New West Technologies); Juliet Newson (International Geothermal Association); Saboth Garg (Leidos Inc.); Chester Weiss (Sandia National Laboratories); Jeff Winick (Schlumberger); and numerous workshop participants for their insights and suggestions relating to this study. We also thank the NREL review team, including Amanda Kolker, Don Gwinner, Kendra Palmer, Scott Gossett, Emily Newes, Jeff Logan, and David Mooney.

The Geothermal Resource Portfolio Optimization and Reporting Technique (GeoRePORT) is a product of the considerable effort of many parties. Since 2012, analysts at NREL, LBNL, and New West Technologies have been deeply involved in research, meetings, outreach, review, and workshops, analyzing and negotiating content to find solutions to divergent views.

The six documents of the GeoRePORT reflect the intellectual contributions of these many players. These end products have been enabled because of a shared vision that this work can make a significant contribution to advancing geothermal deployment. 


\section{LIST OF ACRONYMS}

$\begin{array}{ll}\text { AAS } & \text { atomic absorption spectrometry } \\ \text { DOE } & \text { U.S. Department of Energy } \\ \text { DTS } & \text { distributed temperature sensor } \\ \text { GTO } & \text { Geothermal Technologies Office } \\ \text { ICP-AES } & \text { inductively coupled atomic emission spectroscopy } \\ \text { ICP-MS } & \text { inductively coupled plasma mass spectrometry } \\ \text { LBNL } & \text { Lawrence Berkeley National Laboratory } \\ \text { MT } & \text { magnetotelluric } \\ \text { NCGs } & \text { non-condensable gases } \\ \text { NREL } & \text { National Renewable Energy Laboratory } \\ \text { PTS } & \text { pressure-temperature spinner } \\ \text { TDS } & \text { total dissolved solids } \\ \text { TEM } & \text { transient electromagnetics } \\ \text { TGH } & \text { thermal gradient hole } \\ \text { USGS } & \text { U.S. Geological Survey } \\ \text { VSP } & \text { vertical seismic profiling }\end{array}$




\section{CONTENTS}

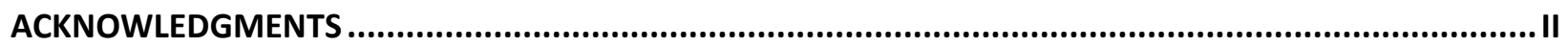

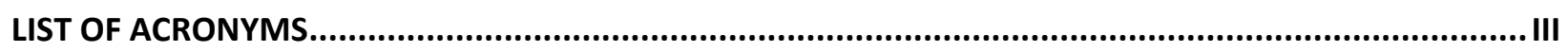

CONTENTS

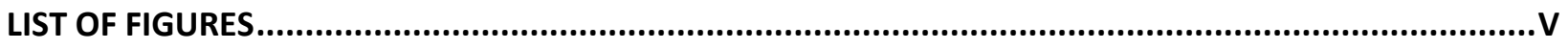

LIST OF TABLES

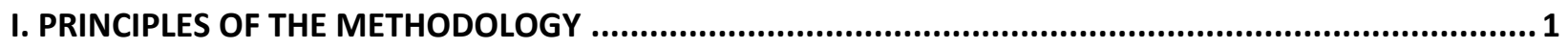

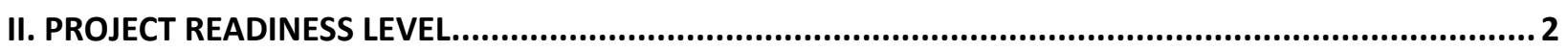

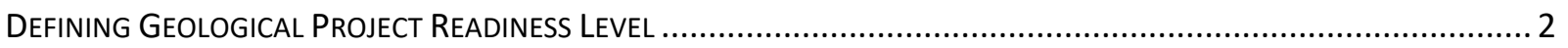

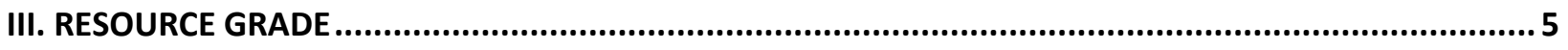

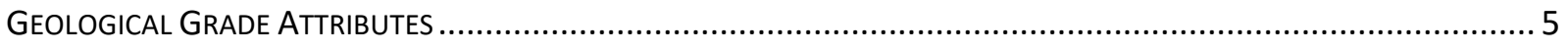

COMPONENTS OF GEOLOGICAL GRADE

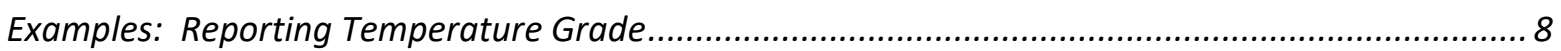

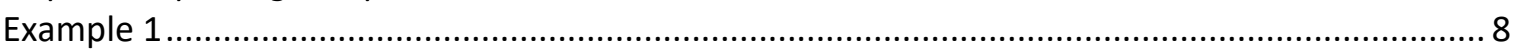

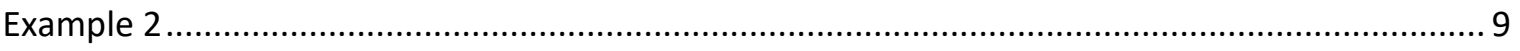

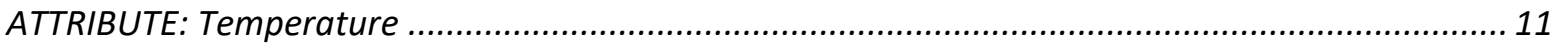

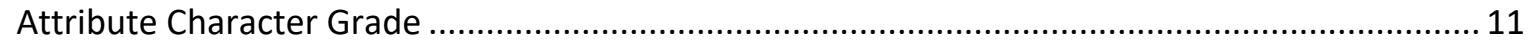

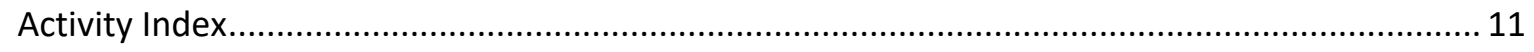

Execution Index

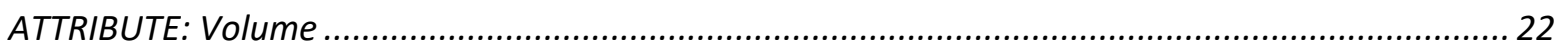

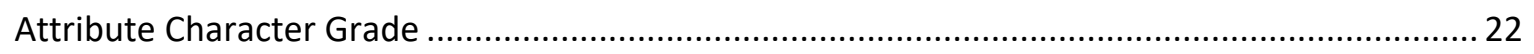

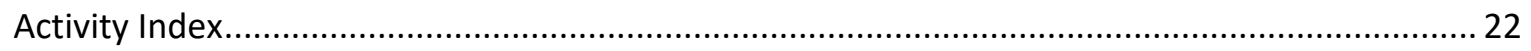

Execution Index

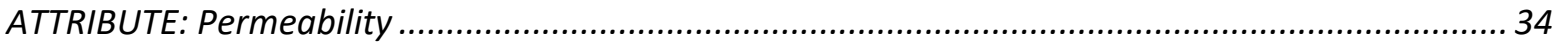

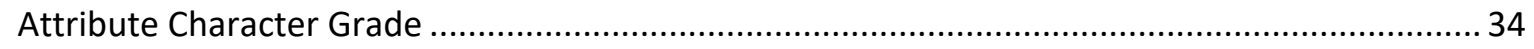

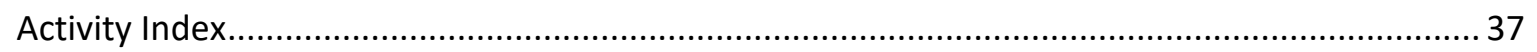

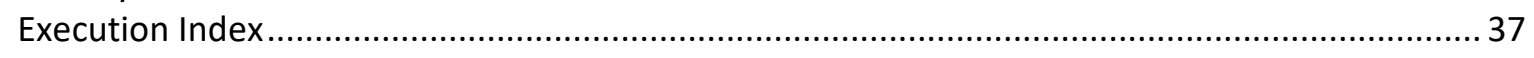

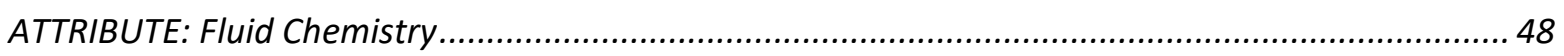

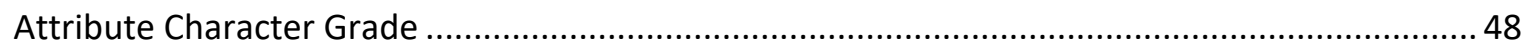

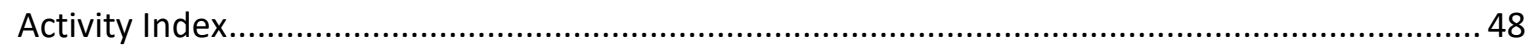

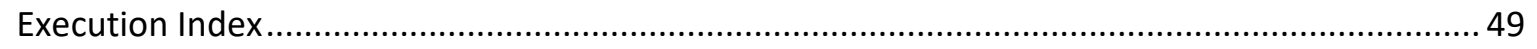

Sub-Attribute Character Grades and Activity Indices Tables .................................................50

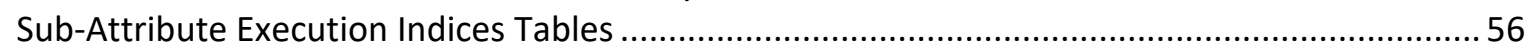

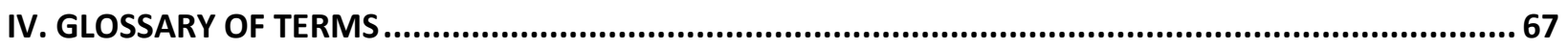

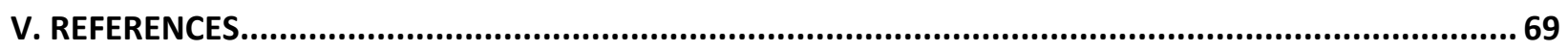

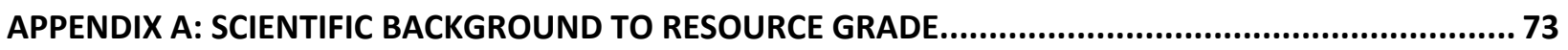




\section{LIST OF FIGURES}

Figure 1. Depiction of geological progress in relation to other forms of project readiness levels.............. 4

Figure 2. Combined Geological Grade Diagram of a Hypothetical Project.............................................. 7

\section{LIST OF TABLES}

Table 1. Qualifying Criteria to Move between Different Geological Project Readiness Levels ................... 3

Table 2. Indices Used to Describe Resource Grades: Character Grade, Activity Index, and

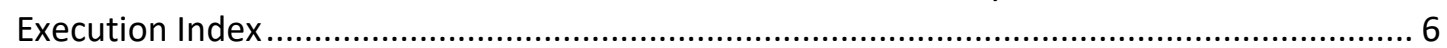

Table 3a. Example of Geothermometry Activities for a Given Project Location ...................................... 8

Table 3b. Example of a Thermal Gradient Activity for a Given Project Location ....................................... 9

Table 4. Character Grade for Temperature Attribute............................................................................. 11

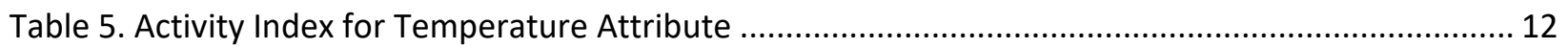

Table 6-1. Execution Index: Downhole Temperature Probe and Thermal Gradient Hole ......................... 14

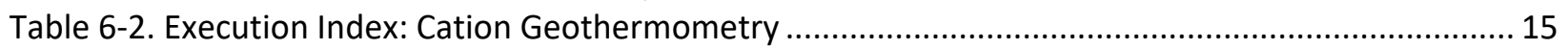

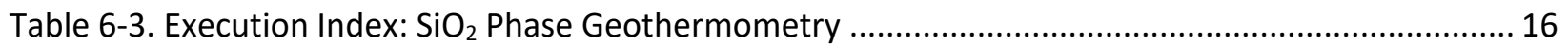

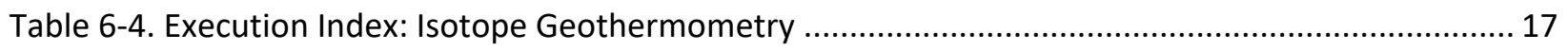

Table 6-5. Execution Index: Multicomponent Geothermometry ........................................................ 18

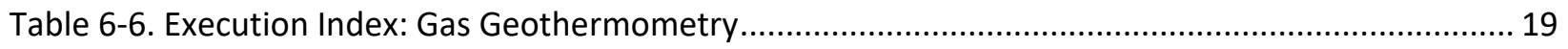

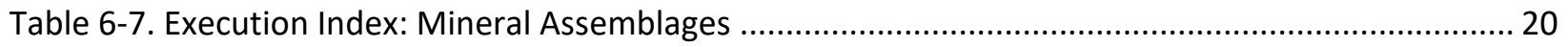

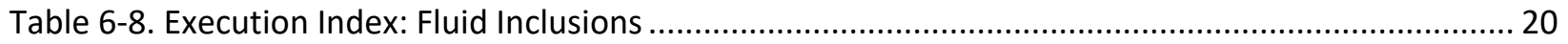

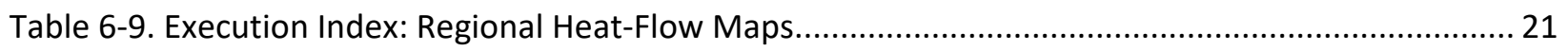

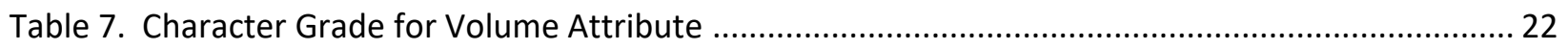

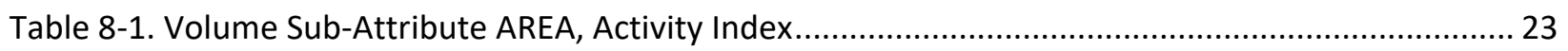

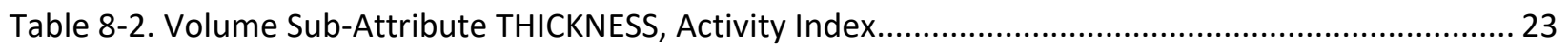

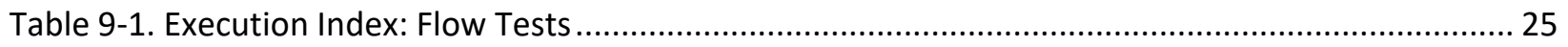

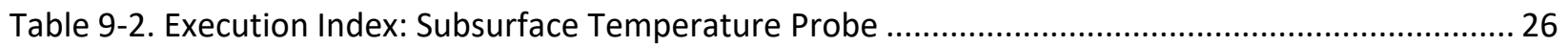

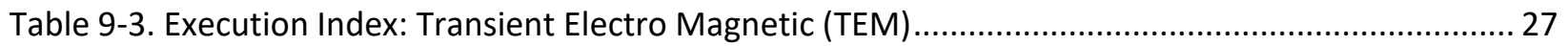

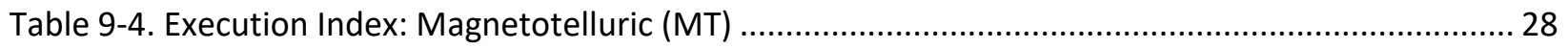

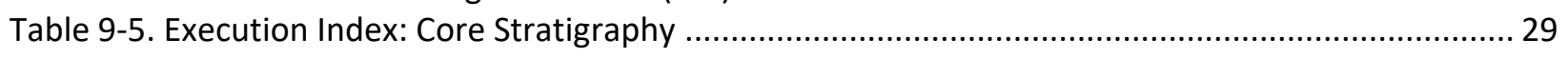

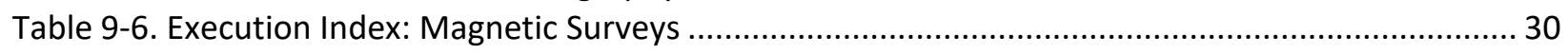

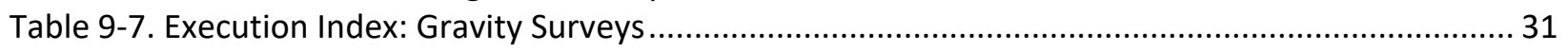

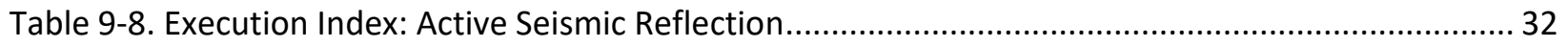

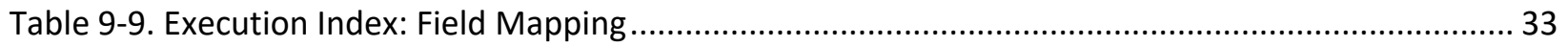

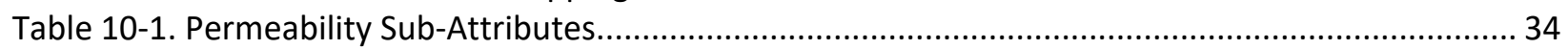

Table 10-2. Permeability Sub-Attribute: Fault/Fracture Orientation ................................................... 35

Table 10-3. Permeability Sub-Attribute: Effective Fault/Fracture Aperture .......................................... 35

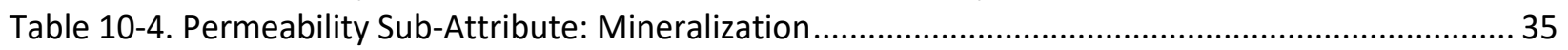

Table 10-5. Permeability Sub-Attribute: Fracture Spacing …........................................................... 36

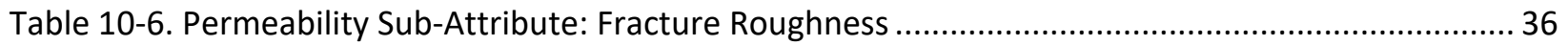

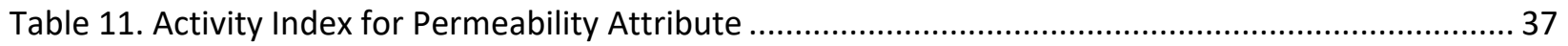

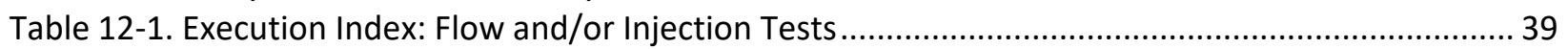

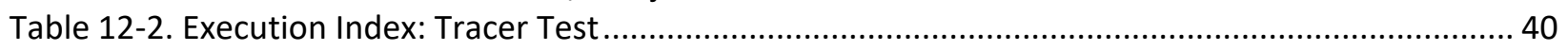

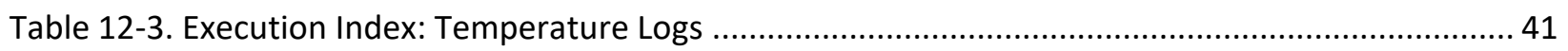

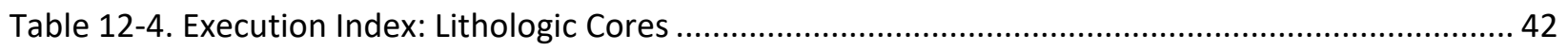




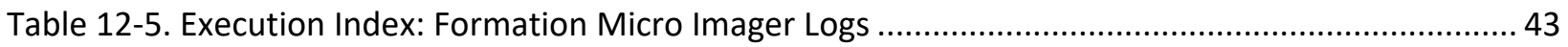

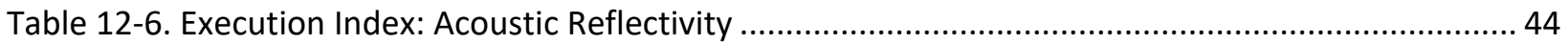

Table 12-7. Execution Index: Vertical Seismic Profiling and Reflection Seismic .......................................45

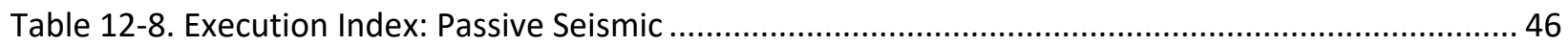

Table 12-9. Execution Index: Field Mapping - Structures and Thermal Features ................................... 47

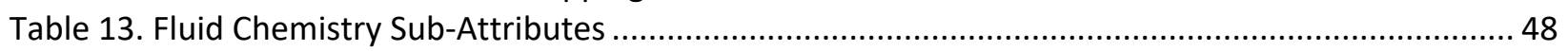

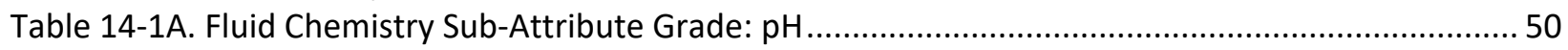

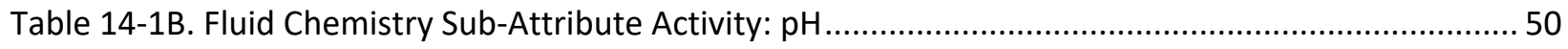

Table 14-2A. Fluid Chemistry Sub-Attribute Grade: Corrosive Gas Content $\left(\mathrm{HCl}, \mathrm{SO}_{2}\right.$, and/or $\left.\mathrm{H}_{2} \mathrm{~S}\right) \ldots \ldots \ldots . . . .51$

Table 14-2B. Fluid Chemistry Sub-Attribute Activity: Corrosive Gas Content ......................................... 51

Table 14-3A. Fluid Chemistry Sub-Attribute Grade: Non-Condensable Gas Content................................ 52

Table 14-3B. Fluid Chemistry Sub-Attribute Activity: Non-Condensable Gas Content............................. 52

Table 14-4A. Fluid Chemistry Sub-Attribute Grade: Bicarbonate Content .............................................5 53

Table 14-4B. Fluid Chemistry Sub-Attribute Activity: Bicarbonate Content............................................ 53

Table 14-5A. Fluid Chemistry Sub-Attribute Grade: Total Dissolved Solid Content .................................5 54

Table 14-5B. Fluid Chemistry Sub-Attribute Activity: Total Dissolved Solid Content .............................. 54

Table 14-6A. Fluid Chemistry Sub-Attribute Grade: Silica Content ...................................................... 55

Table 14-6B. Fluid Chemistry Sub-Attribute Activity: Silica Content .................................................... 55

Table 15-1. Execution Index: Laboratory Analysis - pH .................................................................5

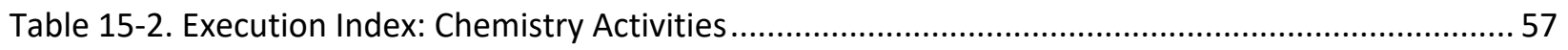

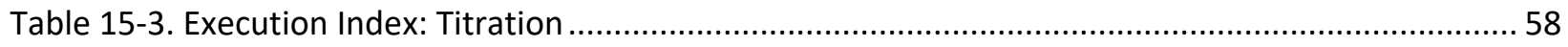

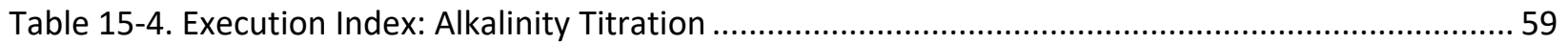

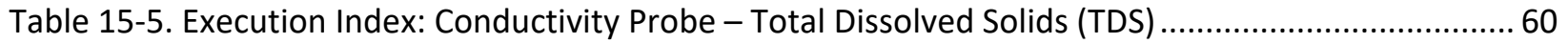

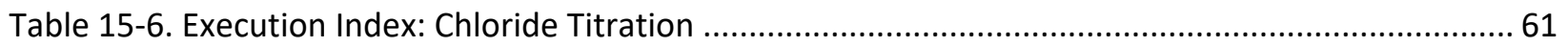

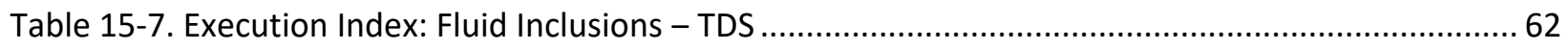

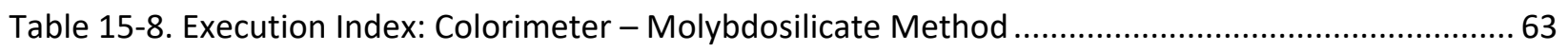

Table 15-9. Execution Index: Inductively Coupled Plasma Atomic Emission Spectroscopy (ICP-AES) ....... 63

Table 15-10. Execution Index: Inductively Coupled Plasma Mass Spectrometry (ICP-MS) .......................64

Table 15-11. Execution Index: Atomic Absorption Spectrometry (AAS) .................................................6 65

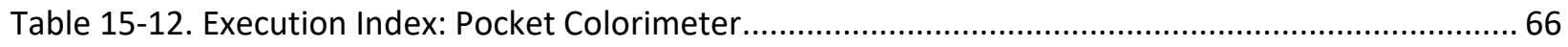




\section{PRINCIPLES OF THE METHODOLOGY}

The Geothermal Resource Portfolio Optimization and Reporting Technique (GeoRePORT) system is based on the concept that a geothermal system can be described both in terms of the quality of the geothermal resource as it relates to the potential to extract heat (resource grade) and the progress of research and development over the lifetime of the project (project readiness level).

By assessing the major characteristics of a geothermal resource and categorizing the techniques used and how well the research technique was implemented, users can report a resource grade. The resource grade covers multiple geological, technological, and socioeconomic attributes that can be compared across play types and geothermal areas. The grade of each resource is intended to be refined, if needed, as new and better information is collected and interpreted.

By assessing the exploration and development activities of the project, users can report on past and planned incremental project readiness levels. Like the resource grade, project readiness level will continually be updated throughout the project lifetime.

Resource grade and project readiness level are reported for three assessment categories: geological, technical, and socioeconomic. Each category has specific criteria and guidelines for assessing both resource grade and project readiness level, as outlined in each of the following assessment tools (and associated colors):

- Geological Assessment Tool (representative color: red)

- Technical Assessment Tool (representative color: blue)

- Socioeconomic Assessment Tool (representative color: green)

Additionally, users may need to estimate the project size (often reported in megawatts-electric [ $\left.\mathrm{MW}_{\mathrm{e}}\right]$ or megawatts-thermal $\left.\left[\mathrm{MW}_{\mathrm{th}}\right]\right)$. To provide consistency in calculating resource potential for comparisons, the protocol is currently developing a separate Resource Size Assessment Tool.

These assessment tools are written for geothermal professionals assigned to report resource grade and project readiness level to the U.S. Department of Energy (DOE). Therefore, it is assumed that:

1. The exploration activities described in this report will be planned, executed, and interpreted by skilled geoscientists.

2. Preparers of reports using the GeoRePORT Protocol are knowledgeable of geothermal systems and the different exploration activities. The guidance in these documents does not replace expertise in preparing, selecting, and interpreting data.

For additional background on the GeoRePORT Protocol, see the Background Document. 


\section{PROJECT READINESS LEVEL}

The GeoRePORT Protocol divides the concept of project readiness levels based on the three assessment categories: geological, technical, and socioeconomic. As projects progress from one development phase to the next, they pass through "activity thresholds"-minimum activities required to qualify for the next level. For each category, numerous qualifying criteria are defined to represent six different levels (0-5) of project readiness: unassessed, undiscovered, inferred, measured, tested, and examined.

\section{DEFINING GEOLOGICAL PROJECT READINESS LEVEL}

The Geological Project Readiness Level's qualifying criteria describe exploration activities at a project location. The qualifying criteria are used to indicate the amount of activity that has occurred in an area and not whether those activities found a geothermal resource. Geological readiness levels are rarely reduced, because, for example: the difference between an undiscovered resource and an inferred resource is the completion of some form of field sampling-once sampling is completed, the project cannot return to the undiscovered category. The associated qualifying criteria for geological project readiness are shown in Table 1: 
Table 1. Qualifying Criteria to Move between Different Geological Project Readiness Levels

\begin{tabular}{|c|c|c|}
\hline \multicolumn{2}{|c|}{$\begin{array}{l}\text { Geological Project } \\
\text { Readiness Level }\end{array}$} & Qualifying Criteria \\
\hline G1 & Undiscovered & $\begin{array}{l}\text { For a resource to be considered "Undiscovered," the potential is estimated by } \\
\text { one of the following activities: } \\
\text { 1. Field mapping-e.g., structural features, hydrothermal alteration surface } \\
\text { manifestations } \\
\text { 2. Shallow heat-flow studies (2-m probe) } \\
\text { 3. Extrapolation of third-party data } \\
\text { 4. Remote sensing }\end{array}$ \\
\hline
\end{tabular}

Field testing/sampling

For a resource to be considered "Inferred," both of the following criteria must be met:

1. Resource temperature is estimated using at least one of the following methods:

\begin{tabular}{l|l|l|l|l|l} 
G2 & a. Well-executed geothermometry \\
b. Thermal gradient holes
\end{tabular}

2. Conceptual model of the system is supported by data from surface geophysical surveys

Slim/core hole into the reservoir

For a resource to be considered "Measured," all of the following criteria must be met:

1. Temperature is measured at the reservoir level using the following methods:

a. Downhole probe in slimhole(s) drilled into the reservoir

G3

Measured 2. Temperature is corroborated using at least one of the following methods:

a. Geothermometry (preferably using well fluid)

b. Assessment of hydrothermal mineral assemblages taken from cores and/or cuttings

Full-diameter well/well test

For a resource to be considered "Tested," all of the following criteria must be met:

1. At least one full-diameter well has been drilled into the reservoir

G4 Tested

2. The reservoir permeability has been evaluated with at least one of the following methods:

a. Flow tests and/or

b. Pressure build-up/draw-down tests

Multiple full-diameter wells drilled

For a resource to be considered "Examined," the following criteria must be met:

G5 Examined 1. Two or more full-scale wells must be drilled and flow tested

The geothermal project readiness level is meant to indicate the amount of activity that has occurred in an area-not whether those activities resulted in the identification of a viable geothermal reservoir. Select all applicable qualifying criteria for the level of geological progress that best describes the exploration activities that have occurred to date. For example, a project that has had extensive geochemistry and geophysical testing (which would qualify as "Measured") that just today finished drilling its first full-diameter well should now be classified as "Tested," regardless of whether the well is producing. In GeoRePORT, the project would continue to be classified as "Tested" even if the next exploration activities performed are additional geophysical tests. The GeoRePORT tool will automatically populate the project readiness level based on the selections.

GeoRePORT recognizes that exploration activities alone cannot describe a viable geothermal resource. In this Protocol, the project readiness level is determined by the combination of the geological, 
technical, and socioeconomic project readiness levels. Figure 1 shows the relationship between these combined project readiness levels. For more information on the Technical and Socioeconomic Progress Readiness Levels, refer to the Background Document and the associated Assessment Tools.

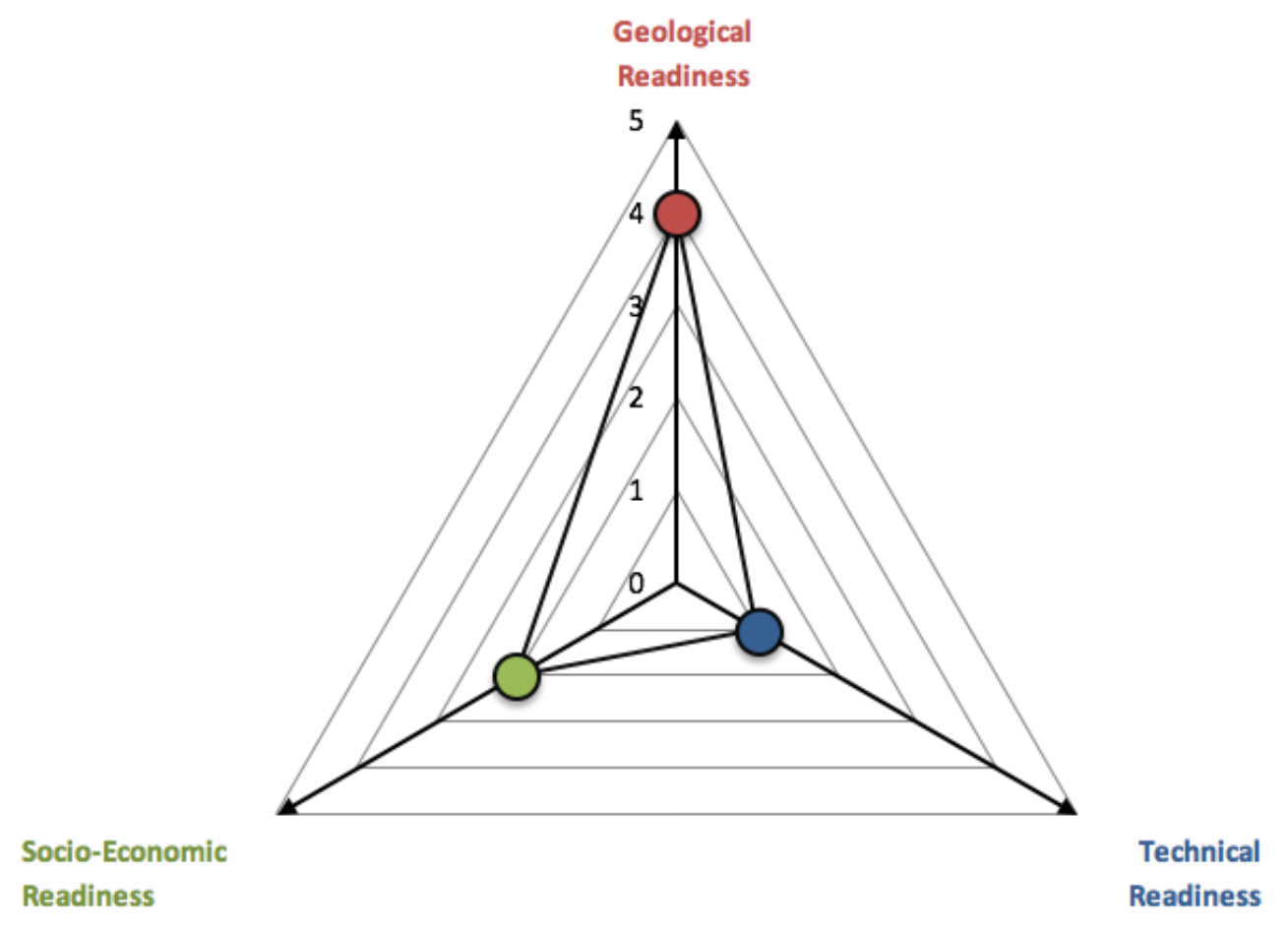

Figure 1. Depiction of geological progress in relation to other forms of project readiness levels 


\section{RESOURCE GRADE}

The attributes used by this Protocol to describe a geothermal resource include constraints on the quality of the geothermal resource as well as the technical and socioeconomic characteristics that determine whether the heat can be produced.

Attributes controlled by the structural, geophysical, geochemical, and hydrological constraints are important in describing the geological grade of a geothermal system. Other attributes important to the feasibility of extracting the geothermal resource due to technical or socioeconomic barriers are addressed in the Technical Assessment Tool and Socioeconomic Assessment Tool documents, respectively.

Each attribute is ranked on a scale of " $A$ " through " $E$," with " $A$ " indicating the highest of the range of values for that attribute. An attribute grade of " $A$ " is not necessarily the "best" value for a specific project goal. Some business models or plant designs may target grades lower than A for some or all of the attributes. Examples are given below:

- Some developers may be targeting average temperature resources (Temperature Grade $=\mathrm{C}$ ) and poor fluid chemistry (Fluid Chemistry Grade $=\mathrm{D}-\mathrm{E}$ ) to take advantage of secondary mineral recovery potential from the geothermal brine.

- Near-field resources (resources located near operating plants) may have high temperatures but low permeability and/or fluid and may be candidates for the application of enhanced geothermal system techniques.

- A very high-temperature resource does not necessarily need to have a large volume to be economical for some business models; in fact, a small- or average-sized, high-temperature resource could be a viable target.

As these examples indicate, each developer must evaluate which grades are appropriate for his or her target business model. Resources with all attribute grades equaling A rarely exist.

\section{GEOLOGICAL GRADE ATTRIBUTES}

Attributes controlled by the structural, geophysical, geochemical, and hydrological constraints are important in describing the geological grade. The usefulness of a geothermal resource can be described by the available work of the geothermal fluid-the thermodynamic exergy. The exergy that can be extracted from the heat of a geothermal reservoir is related to the flow rate of fluid and the difference between the enthalpy of the fluid in the reservoir and at the surface (DiPippo 2004). Using this thermodynamic basis, the following attributes represent the geologic constraints on the quality of the geothermal heat resource:

- Temperature: The in situ reservoir temperature indicates the amount of energy carried by the geothermal fluid, and it is thus a commonly used proxy for the available enthalpy of the fluid.

- Volume: The size of the reservoir (thickness and area/extent) is necessary for determining the quantity of heat available. 
- Permeability: The permeability of the reservoir rock, often driven by the degree to which the formations are fracture-dominated, largely controls the accessibility and potential recovery of the heat.

- Fluid Chemistry: The geothermal brines and/or gases may be so corrosive or deposit such significant scales that specific tools or materials may have to be used, and special treatments may be necessary, which potentially increases project costs.

These characteristics of a geothermal system are best evaluated in the context of a conceptual model that integrates and portrays geological, geophysical, geochemical, and hydrologic data to constrain the critical elements: heat source, permeable flow pathways, reservoir seal, and resource boundaries (Cumming 2009). Conceptual models are updated as new data and interpretations are obtained. Using these conceptual models, more robust numerical reservoir models are then typically developed from natural-state models of exploration activity outcomes.

Refer to the Resource Size Assessment Tool (pending funding for completion) for a more detailed discussion of how these values are chosen and used to estimate heat in place (i.e., $M W_{\text {th }}$ ) and extractable energy that can be utilized for power generation $\left(\mathrm{MW}_{\mathrm{e}}\right)$.

\section{COMPONENTS OF GEOLOGICAL GRADE}

GeoRePORT also considers the activities conducted to understand each attribute, and what is known about the quality of the data collected. The methodology divides each attribute into three separate indices describing distinct features of each attribute, as outlined in Table 2.

Table 2. Indices Used to Describe Resource Grades: Character Grade, Activity Index, and Execution Index

\begin{tabular}{|c|c|c|}
\hline Index & Description & Example \\
\hline $\begin{array}{l}\text { Character } \\
\text { Grade }\end{array}$ & $\begin{array}{l}\text { Describes the character itself-i.e., } \\
\text { what is the intrinsic measurement that } \\
\text { best describes the reservoir? }\end{array}$ & $\begin{array}{l}\text { Is this a high-temperature (Grade A) or low- } \\
\text { temperature (Grade E) resource? }\end{array}$ \\
\hline $\begin{array}{l}\text { Activity } \\
\text { Index }\end{array}$ & $\begin{array}{l}\text { Qualitative ranking of activities used to } \\
\text { assign the character grade appropriate } \\
\text { for each attribute-i.e., how well is the } \\
\text { character grade known? }\end{array}$ & $\begin{array}{l}\text { Do you have a downhole measurement of } \\
\text { reservoir fluids temperatures (Activity A), or did } \\
\text { you estimate the value from a heat-flow map } \\
\text { (Activity E)? }\end{array}$ \\
\hline $\begin{array}{l}\text { Execution } \\
\text { Index }\end{array}$ & $\begin{array}{l}\text { Compares the diligence with which the } \\
\text { activity was executed-i.e., how much } \\
\text { do we know about the quality of } \\
\text { execution of that activity? }\end{array}$ & $\begin{array}{l}\text { If activity is geochemistry, was the appropriate } \\
\text { geothermometer used? Were proper assumptions } \\
\text { made? Were fluids sampled appropriately? Were } \\
\text { cations and anions in balance? }\end{array}$ \\
\hline
\end{tabular}

For each attribute, the character grade describes the current project within the range of possible quantitative and qualitative measurements for a given area. When evaluating a resource's attribute character grade, multiple aspects of the attribute sometimes contribute to its grade. To assess these multiple aspects, sub-attribute indices have been developed for applicable components of the grade. For example, when considering the permeability attribute, we look at several sub-attributes, such as the fault and fracture orientation, effective aperture, mineralization, fracture spacing, and fracture roughness. 
The activity index describes the common activities used to determine the character grade-both directly (measured values) and indirectly (indicated values). The execution index describes how well the activity was implemented. During the exploration process, activities are performed (activity index), the quality of the data is determined (execution index), and the outcome is reported (character grade).

These four attribute grades, and their associated activity and execution indices, are displayed graphically in a polar-area chart (Figure 2). The dark wedges indicate resource grade (What is your resource like?); the light wedges indicate certainty (How much do you trust the data?). For more information, see the Background Document.

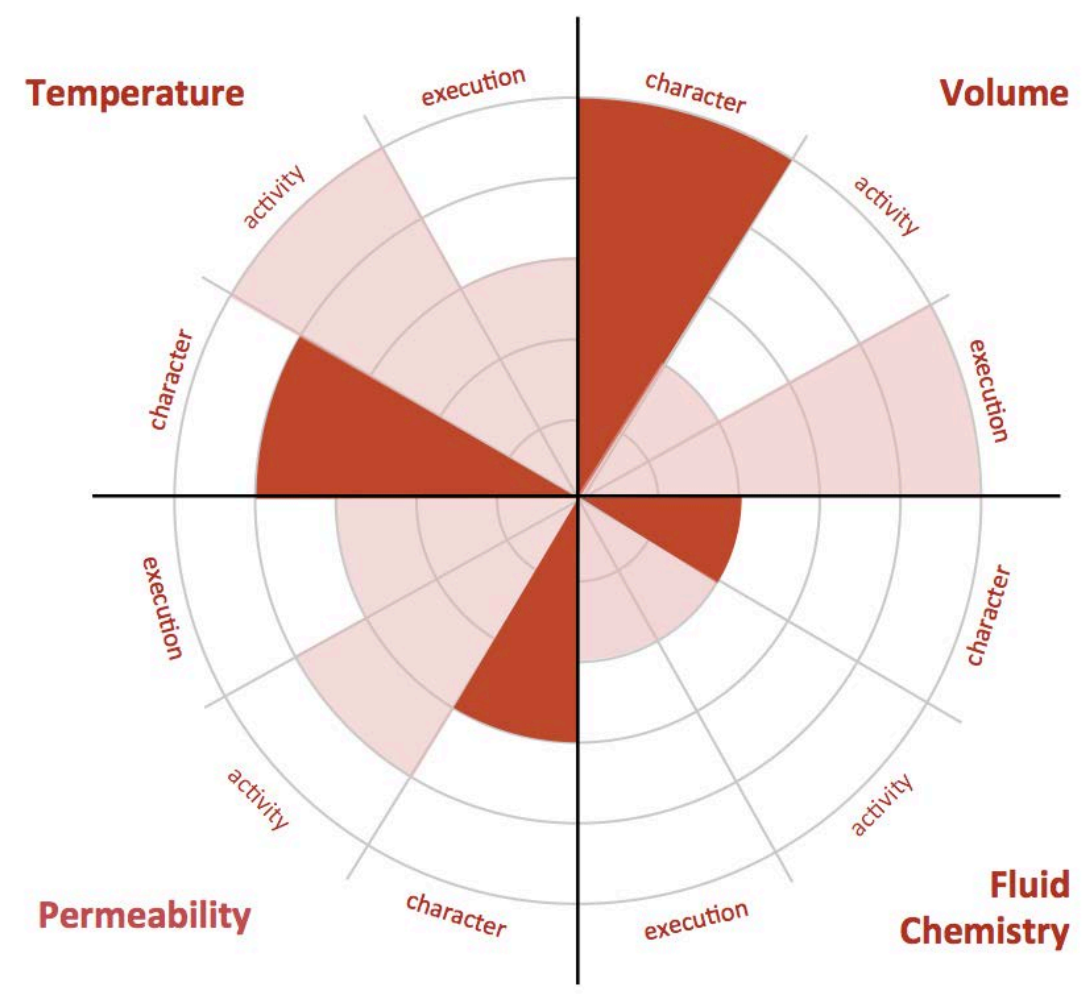

Figure 2. Combined Geological Grade Diagram of a Hypothetical Project

As a reminder, this Protocol was developed to provide consistency among the user community in reporting; it is neither a prescription for conducting exploration and field development, nor a replacement for expertise and conceptual or reservoir models.

Refer to the Technical Assessment Tool and Socioeconomic Assessment Tool Protocol documents for details on the factors relevant to technical and socioeconomic grades. 


\section{EXAMPLES: REPORTING TEMPERATURE GRADE}

We provide an example to aid users in understanding how to use this Protocol to select the most representative geothermal resource temperature value.

Often, multiple temperature estimates are made from different observation points (e.g., different thermal features and wells) and using different methods (e.g., geothermometry, temperature-gradient wells). To better understand the geothermal system, it is important to evaluate data in the context of a conceptual model. For temperature data, some values may be lower (e.g., if measuring temperature of outflow zone) or higher (e.g., if measuring in an upflow zone), depending on the location of the measurement in the context of the conceptual model. The following examples explain how to report when multiple data are available.

\section{Example 1}

The following data are available for a given area:

Table 3a. Example of Geothermometry Activities for a Given Project Location

\begin{tabular}{|c|c|c|c|}
\hline Activity & $\begin{array}{l}\text { Measured/ } \\
\text { Estimated } \\
\text { Temperature }\end{array}$ & $\begin{array}{l}\text { Execution } \\
\text { Comments }\end{array}$ & Notes \\
\hline $\begin{array}{l}\text { Gas } \\
\text { Geothermometry } \\
\text { (gas collected from } \\
\text { springs) }\end{array}$ & $225^{\circ}-275^{\circ} \mathrm{C}$ & $\begin{array}{l}\text { - Used published } \\
\text { geothermometry } \\
\text { data }\end{array}$ & $\begin{array}{l}\text { When gas passes through water, gas reacts } \\
\text { with the water and some of the } \mathrm{H}_{2} \mathrm{~S} \text { and } \mathrm{NH}_{3} \\
\text { is lost. Estimated temperatures are not as } \\
\text { reliable as gas collected from fumaroles. }\end{array}$ \\
\hline $\begin{array}{l}\text { Gas } \\
\text { Geothermometry } \\
\text { (gas collected from } \\
\text { fumaroles) }\end{array}$ & $225^{\circ}-275^{\circ} \mathrm{C}$ & $\begin{array}{l}\text { - Used published } \\
\text { geothermometry } \\
\text { data }\end{array}$ & $\begin{array}{l}\text { When fumaroles have temperatures above } \\
\text { boiling, gases are not being lost. Gas samples } \\
\text { are more representative of the geothermal } \\
\text { reservoir. }\end{array}$ \\
\hline $\begin{array}{l}\text { Thermal Gradient } \\
\text { Hole (TGH) }\end{array}$ & $150^{\circ} \mathrm{C}$ at $600 \mathrm{~m}$ & $\begin{array}{l}\text { - Holes do not } \\
\text { reach the } \\
\text { reservoir } \\
\text { - Temperature } \\
\text { measured } 2 \text { hours } \\
\text { after drilling }\end{array}$ & $\begin{array}{l}\text { We assume TGH data provide a minimum } \\
\text { reservoir temperature (unless there is a } \\
\text { thermal gradient reversal). Horner plot } \\
\text { analysis can be used to project equilibrated } \\
\text { temperatures from multiple temperature } \\
\text { readings. However, because the temperature } \\
\text { measurements were taken so soon after } \\
\text { drilling, we assume the measurement was not } \\
\text { an equilibrated temperature. Measured } \\
\text { temperature in the TGH is likely lower than } \\
\text { actual formation temperature. }\end{array}$ \\
\hline
\end{tabular}

No liquid geothermometry was conducted on these bicarbonate springs because temperatures would not be representative of the reservoir. These waters often represent mixtures of surface waters mixed with steam and $\mathrm{CO}_{2}$, and thus did not equilibrate under reservoir conditions.

As part of developing the conceptual model, the expert geologist uses geophysical data to estimate the depth of the reservoir. The thermal gradient holes (TGHs) help to constrain the lower limits of temperature, but also help to constrain the locations of the upflow and the outflow zones. The expert then extrapolates the thermal gradient from the TGH wells down to the reservoir $(1.5-2 \mathrm{~km})$ in the location of the upflow zones and obtains a temperature of $250^{\circ} \mathrm{C}$. This is consistent with the data from 
the gas geothermometry. The expert would want to report the estimated temperature of the upflow and not the outflow zone.

The expert geologist reports:

Temperature Grade:

Activity Index:

Execution Index:
B $\quad 230^{\circ}-300^{\circ} \mathrm{C}($ Table 4$)$

B Estimated temperatures: Geothermometry (geothermal brines and gases) (Table 5)

C Results taken from previous third-party studies of the area (either literature or contractors) with little or limited information on survey methods, replication, or error (Table 6-1)

\section{Example 2}

The following data are available for a given area:

Table 3b. Example of a Thermal Gradient Activity for a Given Project Location

\begin{tabular}{|l|l|l|l|}
\hline \multicolumn{1}{|c|}{ Activity } & $\begin{array}{c}\text { Measured/Estimated } \\
\text { Temperature }\end{array}$ & $\begin{array}{c}\text { Execution } \\
\text { Comments }\end{array}$ & \multicolumn{1}{c|}{ Notes } \\
\hline $\begin{array}{l}\text { Thermal Gradient } \\
\text { Hole }\end{array}$ & $150^{\circ} \mathrm{C}$ at $1,200 \mathrm{~m}$ & $\begin{array}{l}\text { - Hole does not } \\
\text { reach the } \\
\text { reservoir } \\
\text { Temperature } \\
\text { probe allowed to } \\
\text { equilibrate }\end{array}$ & $\begin{array}{l}\text { We assume TGH data provide a } \\
\text { minimum reservoir temperature } \\
\text { (unless there is a thermal gradient } \\
\text { reversal). }\end{array}$ \\
& & & \\
\hline
\end{tabular}

The prevailing conceptual models in this area suggest that these TGHs were drilled on the margins of the geothermal system. The expert geologist expects that the thermal gradient (and reservoir temperatures) in the location of these holes is lower than in the central portion of the reservoir near the upflow zone. The conceptual model suggests that the upflow zone, however, is inside a nearby national park where development is not allowed. Additionally, there is evidence that fluids in the volcanic upflow portion of the system are highly corrosive. Analogues in the Philippines with similar vapor-rich, acidic cores have neutralized systems along the margins that have high enough temperatures to make them economically feasible to develop. In this case, the expert would choose to report estimated temperatures of the outflow zone (and not the upflow zone) because this is where planned development will take place. 
The expert geologist reports:

Temperature Grade:

Activity Index:

Execution Index:
C $\quad 150^{\circ}-230^{\circ} \mathrm{C}$ (Table 4$)$

D Extrapolated temperature: TGH/well(s) (Table 5)

A Meets the criteria below (Table 6-1)

- The analytical quality of results can be shown to be high (based on sampling replication and instrument calibration logs).

- Reconnaissance was completed both along known faults and thermal features as well as externally to active surface features and thermal areas.

- Geophysical data and/or other geological knowledge have clearly identified the depth of the top of the reservoir.

- Results are from stabilized logs, not initial readings.

- The TGH is drilled as deep as possible to reduce the distance of extrapolation.

- Temperature-depth logs are continuous.

- Knowledge of local geology/fault structure exists for the entire log depth. 


\section{ATTRIBUTE: TEMPERATURE}

\section{Attribute Character Grade}

Although enthalpy is the most relevant thermodynamic characteristic in describing the available heat of a reservoir, enthalpy cannot be measured directly. Instead, the in situ temperature of the geothermal fluid is used because it represents the heat available to be transferred. Many prior geothermal classification systems have used temperature ranges (Muffler and Cataldi 1978; Hochstein 1988; Bendritter and Cormy 1990; Nicholson 1993; Axelsson and Gunnlaugssen 2000; Kaya et al. 2011). The temperature grades in this Protocol are loosely based on Sanyal's (2005) distinctions among the range of possible temperatures into five categories, shown in Table 4. These values refer to the reservoir temperature and phase(s) within the geothermal system, not the geothermal fluid measured at a wellhead. Ambient temperature is discussed in this Protocol's Resource Size Assessment Tool.

Table 4. Character Grade for Temperature Attribute

\begin{tabular}{|c|c|c|}
\hline Grade & Value Range & Description \\
\hline A & $\begin{array}{l}\geq 300^{\circ} \mathrm{C} \\
\text { or steam }\end{array}$ & $\begin{array}{l}\text { High-temperature, two-phase, liquid-dominated } \\
\text { OR high-enthalpy vapor-dominated }\end{array}$ \\
\hline B & $230-<300^{\circ} \mathrm{C}$ & $\begin{array}{l}\text { Two-phase, liquid-dominated systems: } \\
\text { - High-temperature, high-enthalpy } \\
\text { - Moderate-temperature, moderate-enthalpy }\end{array}$ \\
\hline C & $150-<230^{\circ} \mathrm{C}$ & $\begin{array}{l}\text { Moderate- to low-temperature, moderate- to low- } \\
\text { enthalpy liquid-only systems }\end{array}$ \\
\hline D & $90-<150^{\circ} \mathrm{C}$ & Low-temperature systems \\
\hline E & $<90^{\circ} \mathrm{C}$ & Very low-temperature systems \\
\hline
\end{tabular}

As shown in Table 4, a resource with a high temperature $\left(\geq 300^{\circ} \mathrm{C}\right)$ or that is steam-dominated is assigned a temperature character grade of $A$, whereas a resource with a low temperature $\left(<90^{\circ} \mathrm{C}\right)$ is assigned a temperature character grade of $\mathrm{E}$.

To report temperature grade, select the temperature value obtained from the most reliable activity (technique), taken in the context of the conceptual model for the system. Having multiple methods that provide a similar result increases the likelihood that the temperature value selected is reliable. Alternatively, use modeled temperatures from a comprehensive reservoir model to select the temperature grade.

For further guidance on choosing the most likely, upper, and lower bounds of temperature estimates, refer to Section I (Selecting Estimates of Reservoir Temperature) in the Resource Size Assessment Tool.

\section{Activity Index}

Research and exploration methods to evaluate resource temperature fall into two general areas: geothermometry and downhole temperature measurements obtained from drill holes. Exploration programs will likely use more than one activity for estimating temperature. Remote sensing or surface mapping of hydrothermal manifestations provide a minimum temperature estimate; but because these techniques are heavily influenced by thermal features, these methods are not reliable constraints on reservoir temperature. 
Table 5 lists the activities identified and their assigned index values, ranked by the likelihood that the data collected from these activities represent the actual reservoir temperature (in most cases).

Table 5. Activity Index for Temperature Attribute

\begin{tabular}{|c|c|c|}
\hline Index & Activity & $\begin{array}{l}\text { See Related } \\
\text { Execution Index }\end{array}$ \\
\hline A & $\begin{array}{l}\text { Measured temperatures: Downhole temperature probe readings } \\
\text { (well(s) drilled into reservoir) }\end{array}$ & Table 6-1 \\
\hline B & $\begin{array}{l}\text { Estimated temperatures: Geothermometry (geothermal brines } \\
\text { and gases) }\end{array}$ & Tables 6-2 to 6-6 \\
\hline C & $\begin{array}{l}\text { Estimated temperatures: Geothermometry (immature or mixed } \\
\text { fluids, inconsistent results between geothermometers); alteration } \\
\text { mineral assemblages; fluid-inclusion homogenization } \\
\text { temperatures }\end{array}$ & Tables $6-2$ to $6-8$ \\
\hline D & Extrapolated temperature: Thermal gradient hole (TGH)/well(s) & Table 6-1 \\
\hline E & Extrapolated temperature: Regional heat-flow data & Table 6-9 \\
\hline
\end{tabular}

When selecting the appropriate activity index, consider the conceptual and/or reservoir model so as to choose the most representative reservoir temperature measurement(s). If measurement results are inconsistent with the conceptual model, then select the activity index that corresponds to the activity(ies) that was/were performed with the highest quality, i.e., their data quality corresponds to an execution index of A or B (and consider reevaluating the conceptual model).

If there is scientific reason to report other than as prescribed, it is important to note this discrepancy and the justification when reporting.

Refer to the Resource Size Assessment Tool for further guidance in selecting the activity index value that corresponds to the most likely, upper, and lower bounds of temperature measurements.

\section{Execution Index}

For each activity used to measure temperature, the execution indices reflect how the data were verified relative to best practices. The index qualitatively indicates relative error and uncertainty in temperaturerelated exploration activities.

The following tables list the primary types of activity used to measure temperature. ${ }^{1}$ Two important things should be kept in mind:

- These tables are not an exhaustive list of all possible analytical methods that could be used to determine (or estimate) temperature. Those who have used (or wish to use) an analytical

\footnotetext{
${ }^{1}$ References that provide additional details on the uncertainties involved in these techniques include Henley et al. 1984; Giggenbach and Goguel 1989, and Fournier 1991.
} 
method or technique not listed here should contact the DOE Geothermal Technologies Office (GTO) for guidance to create an appropriate execution index for reporting.

- The criteria in these tables are not meant to be guidelines to perform these exploration activities, and they are not an exhaustive list of all quality controls that must be performed. It is assumed that all analyses are performed by an experienced professional. These tables are for reporting purposes only.

For the selected "most-representative" activity used to report temperature grade, select the execution index that best corresponds to the quality of information obtained.

Example (as shown previously): If temperature was determined from gas geothermometry, use Table 6-6 to determine the appropriate execution index (i.e., indicate how well the gas geothermometry was conducted). If information obtained by a contractor performing the TGH can be shown to closely meet the criteria in " $D$," then report the execution index value as " $D$. ." 
Table 6-1. Execution Index: Downhole Temperature Probe and Thermal Gradient Hole

Corresponds to activity index: $A, D$ (Table 5)

Index Execution Details for Downhole Temperature Probes and Thermal Gradient Holes

A - Probe allowed to equilibrate with the wellbore fluids.

- Borehole has equilibrated with the surrounding formation.

- Temperature log run under static conditions.

- Cuttings and/or geophysics confirms measurement within the reservoir (i.e., downhole alteration mineralogy consistent with reading).

- Repeated surveys at the same well/location.

- Frequent calibrations completed that follow a prescribed set of procedures.

- Analytical quality of results can be shown to be high (based on sampling replication and instrument calibration logs).

- Borehole is drilled as deep as possible to reduce distance of extrapolation.

- Temperature-depth logs are continuous.

- Knowledge of local geology/fault structure exists for the entire log depth.

B - Probe allowed to equilibrate with the wellbore fluids or is from a series of temperature measurements with the use of Horner plots.

- Borehole has not equilibrated with the surrounding formation (i.e., drilled recently).

- Temperature log run under flowing conditions.

- Cuttings and/or geophysics have not confirmed measurement within the reservoir (i.e., downhole alteration mineralogy not consistent with readings), but geophysical data and/or other geological knowledge have identified reservoir formations.

- Single survey at the well/location.

- Frequent calibrations completed, but prescribed set of procedures are not consistently followed.

- Borehole is drilled as a moderately deep hole (e.g., $500 \mathrm{~m}$ deep).

- Temperature-depth logs are continuous.

- Knowledge of local geology/fault structure exists for some of the log depth.

C - Results taken from previous third-party studies of the area (either literature or contractors) with little or limited information on survey methods, replication, or error.

D Probe not allowed to equilibrate with the wellbore fluids.

- Borehole has not equilibrated with the surrounding formation (i.e., drilled recently).

- Unknown whether temperature log run was under static or flowing conditions.

- Cuttings and/or geophysics have not confirmed measurement within the reservoir.

- Calibrations are not completed regularly, and no prescribed set of procedures exist.

- Linear extrapolation suggests anomalous temperatures in comparison to nearby locations (i.e., conflicting gradients).

- Minimal TGHs placed given the complexity of underlying geology-and none outside thermal features.

- Boreholes show indications of temperature reversals with depth.

- Temperature-depth logs are not continuous.

- Knowledge of local geology/fault structure does not exist.

E Assumed from studies of analogous geothermal settings or extrapolated from studies of nearby areas. 
Table 6-2. Execution Index: Cation Geothermometry

Corresponds to activity index: $B, C$ (Table 5)

Index Execution Details for Geothermometry: Cation

A - Water composition is appropriate for the geothermometer.

- Chemistry has been evaluated for mixing and boiling relationships, and appropriate corrections are made to determine end members when fluid is known to have mixed with other water sources (e.g., seawater, nonthermal saline brine, or dilute shallow groundwater).

- Interpreted in combination with high-quality data on other physical parameters $(\mathrm{pH}$, dissolved gases).

- Multiple-cation geothermometer systems (e.g., $\mathrm{Na}-\mathrm{K}, \mathrm{Na}-\mathrm{K}-\mathrm{Ca}-\mathrm{Mg}$, K-Mg, and/or Li-Mg) used to corroborate results.

- Cation and anion balance shows minimal gap in vast majority of samples (within $5 \%$ of charge balance).

- Fluid mixing from multiple well feed points does not exist, or is known and addressed.

B - Appropriate corrections are made to determine end members when fluid is known to have mixed with other water sources (e.g., seawater, nonthermal saline brine, or dilute shallow groundwater).

- Appropriate selection of reaction systems (e.g., Na-K-Ca: separate equations for $<100{ }^{\circ} \mathrm{C}$ and $\left.>100{ }^{\circ} \mathrm{C}\right)$.

- Multiple cation geothermometer systems (e.g., Na-K, Na-K-Ca-Mg, K-Mg, and/or Li-Mg) used to corroborate results.

- Fluid mixing from multiple well feed points not known.

C - Results taken from previous third-party studies of the area (either literature or contractors) with little or limited information on survey methods, replication, or error.

D - No (or limited) corrections made even if:

- fluid is known to have mixed with other water sources, or

- partial pressures of $\mathrm{CO}_{2}$ and calcite precipitation are significant.

- Inappropriate application to bicarbonate or acid sulfate waters that are derived from steam heating of near-surface waters and interaction with geothermal gases, and where the fluid cation chemistry does not reflect equilibrium with minerals at reservoir conditions.

- Cation and anion balance shows significant gap in majority of samples, without a functional explanation.

- Fluid mixing from multiple well feed points known, and not addressed.

E Assumed from studies of analogous geothermal settings or extrapolated from studies of nearby areas. 
Table 6-3. Execution Index: $\mathrm{SiO}_{2}$ Phase Geothermometry

Index Execution Details for Geothermometry: $\mathrm{SiO}_{2}$ Phases

A - Corrections for the following effects:

- pH effects on silica solubility when $\mathrm{pH}>9$.

- Salinity effects corrected for waters with higher total dissolved solids (TDS) than seawater.

- Mixing /dilution effects with other sources (groundwater or surface).

- Use of maximum steam loss equation if steam loss is expected from sampled feature.

- Concentrations plotted against enthalpy to confirm appropriate phase selection: (e.g. for silica: amorphous: $<180^{\circ} \mathrm{C}$, chalcedony or quartz: $200^{\circ}-300^{\circ} \mathrm{C}$, quartz: $>300^{\circ} \mathrm{C}$ ).

- Analytical quality of results can be shown to be high (based on standards measured, sample replication, and calibration logs).

- Samples collected appropriately (either diluted or acidified) to prevent silica precipitation.

B - All of the data correction best practices listed above.

- Not plotted against enthalpy.

- Appropriate phase selection.

C - Results taken from previous third-party studies of the area (either literature or contractors) with little or limited information on survey methods, replication, or error.

D - Not enough information available to implement data correction best practices (e.g., erroneous $\mathrm{pH}$, not enough information to identify dissolved silica or proportion of steam separated).

- Significant, unexplained differences in sample results.

- Not plotted against enthalpy.

- Possibly inappropriate phase selection(Sample not collected using appropriate methods-may have had silica precipitation, resulting in lower than expected values.

E Assumed from studies of analogous geothermal settings or extrapolated from studies of nearby areas. 
Table 6-4. Execution Index: Isotope Geothermometry

Corresponds to activity index: $B, C$ (Table 5)

Index Execution Details for Geothermometry: Stable Isotopes

A - Multiple isotope systems (hydrogen, carbon, oxygen, and/or sulfur) provide narrowly constrained temperature based on calculated equilibrium values.

- Analytical quality of results can be shown to be high (based on standards measured, sample replication, and calibration logs).

- Steam and water discharge samples are both collected without air contamination.

B - Some, but not all, of multiple isotope systems (hydrogen, carbon, oxygen, and/or sulfur) provide similar temperatures.

- Corrections can be made for mixing/dilution effects with other water sources (groundwater or surface)-particularly relevant for oxidation of $\mathrm{H}_{2} \mathrm{~S}$ and sulfur-oxidizing bacteria.

C - Results taken from previous third-party studies of the area (either literature or contractors) with little or limited information on survey methods, replication, or error.

D Multiple isotope systems (hydrogen, carbon, oxygen, and/or sulfur) do not yield consistent temperature estimates.

- Mixing/dilution effects with other water sources are not well understood or corrected for.

- Steam and water discharge are not separated completely, or have evidence of air contamination.

E - Assumed from studies of analogous geothermal settings or extrapolated from studies of nearby areas. 
Table 6-5. Execution Index: Multicomponent Geothermometry

Corresponds to activity index: $B, C$ (Table 5)

\section{Index Execution Details for Geothermometry: Multicomponent}

A - Complete liquid and gas analyses, including $\mathrm{Si}, \mathrm{Al}, \mathrm{Mg}$, and Fe.

- Selection of suite of minerals that have equilibrated with the geothermal fluids based on complementary analyses on geologic setting and/or reservoir petrology.

- Optimization captures key processes that may have affected fluid compositions (e.g., boiling, degassing, mixing).

- Uses appropriate thermodynamic database.

- Interpreted in combination with high-quality data on other physical parameters $(\mathrm{pH}$, dissolved gases).

- Multiple geothermometers used to corroborate results.

- Cation and anion balance shows minimal gap in majority of samples.

B - Complete liquid analyses (see above), but no gas measurements.

- Assignment of specific mineral phases to control Al and Fe solubility.

- Assumption of suite of minerals that have equilibrated with the geothermal fluids based on similar geologic settings and/or reservoir petrology.

- Appropriate corrections are made to determine end members when fluid is known to have mixed with other water sources.

- Optimization captures some but not all processes that may have affected fluid compositions (e.g., boiling, degassing, mixing).

- Any $\mathrm{CO}_{2}$ loss can be constrained accurately.

- Uses appropriate thermodynamic database.

- Cation and anion balance shows minimal gap in majority of samples.

C - Results taken from previous third-party studies of the area (either literature or contractors) with little or limited information on survey methods, replication, or error.

D Cation and anion balance shows significant gap in majority of samples, without a functional explanation.

- Application of a standard suite of minerals without any recognition of the appropriate geologic setting (e.g., use of alteration suite for volcanic-hosted system when reservoir rocks consist of altered sedimentary rocks).

- No (or limited) optimization made even if:

- fluid is known to have mixed with other water sources, or

- partial pressures of $\mathrm{CO}_{2}$ and calcite precipitation are significant, or

- uses a default thermodynamic database.

E - Assumed from studies of analogous geothermal settings, or extrapolated from studies of nearby areas. 
Table 6-6. Execution Index: Gas Geothermometry

Corresponds to activity index: $B, C$ (Table 5)

\section{Index Execution Details for Geothermometry: Gas}

A - High-quality samples (well gas separates, fumaroles).

- Appropriate sampling methods used to minimize air contamination.

- Sampling minimizes interaction with shallow fluids.

- Samples from high-flow, high-temperature (superheated) vents.

- Analyses of all of the following gases:

- $\mathrm{H}_{2} \mathrm{~S}$ and $\mathrm{SO}_{2}$

- $\mathrm{CO}$

$-\mathrm{CO}_{2}$

$-\mathrm{CH}_{4}$

$-\mathrm{N}_{2}$

- $\mathrm{Ar}$

- $\mathrm{O}_{2}$

$-\mathrm{NH}_{3}$

- $\mathrm{H}_{2}$

- Gas grid geothermometer employed.

- Assumption of $\mathrm{R}_{\mathrm{h}}$ value of -2.8 is appropriate based on $\mathrm{FeO}-\mathrm{Fe}_{2} \mathrm{O}_{3}$.stability determinations.

B - Sampling completed only from fumaroles and springs (not wells).

- Appropriate sampling methods used to minimize air contamination.

- Sampling minimizes interaction with shallow fluids.

- Samples from high-temperature (superheated) vents with low flow.

- Complete analyses of some, but not all, of the following:

- $\mathrm{H}_{2} \mathrm{~S}$ and $\mathrm{SO}_{2}$

- $\mathrm{CO}$

$-\mathrm{CO}_{2}$

$-\mathrm{CH}_{4}$

- $\mathrm{N}_{2}$

- $\mathrm{Ar}$

- $\mathrm{O}_{2}$

$-\mathrm{NH}_{3}$

- $\mathrm{H}_{2}$

- Assumption of $R_{h}$ value of -2.8 without confirmation that this is appropriate.

C Results taken from previous third-party studies of the area (either literature or contractors) with little or limited information on survey methods, replication, or error.

D - Sampling completed from bubbling springs.

- Air contamination in some samples.

- Samples indicate some interaction with shallow fluids (depletion of gas sulfur species due to formation of dissolved sulfate, dissolution of ammonia into water phase).

- Samples have diffuse flow and have temperatures at or below boiling.

E - Assumed from studies of analogous geothermal settings, or extrapolated from studies of nearby areas. 
Table 6-7. Execution Index: Mineral Assemblages

Corresponds to activity index: $B, C$ (Table 5)

\section{Index Execution Details for Mineral Assemblages}

A - Mineralogy of multiple core samples/thin sections display similar hydrothermal mineral suites, and alteration appears to be latest stage.

B - Mineralogy of core samples/thin sections show similar hydrothermal mineral suites with at least one episode of significant hydrothermal alteration.

C - Results taken from previous third-party studies of the area (either literature or contractors) with little or limited information on survey methods, replication, or error.

D - Mineralogy of core samples/thin sections show hydrothermal mineral suites with significantly different temperature ranges and/or multiple episodes of hydrothermal alteration, none of which match the present system.

- Alteration mineralogy not consistent with fluid chemistry from well and/or measured temperatures (may reflect relict hydrothermal activity).

E Assumed from studies of analogous geothermal settings, or extrapolated from studies of nearby areas.

Table 6-8. Execution Index: Fluid Inclusions

Corresponds to activity index: C (Table 5)

\section{Index Execution Details for Fluid Inclusions}

A - Fluid inclusions hosted by minerals found in multiple core samples/thin sections with a similar hydrothermal mineral suite, and alteration appears to be latest stage.

- Heating-freezing table is appropriately and regularly calibrated for geothermal fluid ranges (daily or weekly, depending on frequency of use).

- Temperature increases $/$ decreases are performed gradually (i.e., $0.1^{\circ}-0.2^{\circ} \mathrm{C} / \mathrm{min}$ ) for high resolution.

- Multiple large inclusions are used in analysis.

- Results create reproducible conclusions.

- Results do not produce multiple populations.

B - Fluid inclusions hosted by minerals found in core samples/thin sections with a similar hydrothermal mineral suite with at least one episode of significant hydrothermal alteration.

- Heating-freezing table is appropriately calibrated for geothermal fluid ranges, but not recently (e.g., not within the recent 6 months).

- Temperature increases/decreases are performed gradually (i.e., $0.1^{\circ}-0.2^{\circ} \mathrm{C} / \mathrm{min}$ ) only when nearing target temperature ranges, and are otherwise $0.5^{\circ} \mathrm{C} / \mathrm{min}$.

- Most inclusions (>50\%) are too small for measurement, but some (>25\%) can be analyzed.

- Results create reproducible conclusions, with few outliers.

C - Results taken from previous third-party studies of the area (either literature or contractors) with little or limited information on survey methods, replication, or error.

D - Fluid inclusions hosted by minerals found in core samples/thin sections with dissimilar mineral suites (i.e., significantly different temperature ranges) with multiple episodes of hydrothermal alteration (and multiple fluid inclusion populations), none of which match the present system.

- Alteration mineralogy not consistent with fluid chemistry from well (may reflect relict hydrothermal activity).

- Heating-freezing table is not calibrated for geothermal fluid ranges.

- Temperature increases/decreases are not performed gradually (i.e., $>0.5^{\circ} \mathrm{C} / \mathrm{min}$ ) and do not target the desired temperature range.

- Most inclusions (>75\%) are too small for measurement or have leaked their gas phase.

- Results suggest multiple populations or are inconclusive.

E - Assumed or extrapolated from studies of analogous geothermal settings or nearby areas. 
Table 6-9. Execution Index: Regional Heat-Flow Maps

Corresponds to activity Index: $E$ (Table 5)

\section{Index Execution Details for Regional Heat-Flow Maps}

A - Proximity of mapping resolution to actual calibration points is high enough to constrain the area of interest.

- Density of actual data coverage behind heat-flow contours is known, and relatively dense.

- Maps corrected for shallow, cold features that overlay deeper heat-flow signal.

- Underlying well bottom-hole temperature data use measured thermal conductivity and $>75 \%$ are equilibrated measurements.

B - Proximity of mapping resolution to actual calibration points is high enough to constrain most boundaries of the area of interest.

- Density of actual data coverage behind heat-flow contours is known, but sparse.

- Maps corrected for major (but not all) shallow, cold features that overly the deeper heat-flow signal.

- Underlying well bottom-hole temperature data use measured thermal conductivity but $<75 \%$ are equilibrated measurements.

\footnotetext{
C - Results taken from previous third-party studies of the area (either literature or contractors) with little or limited information on survey methods, replication, or error.

D Proximity of mapping resolution to actual calibration points is too low to constrain boundaries in the area of interest.

- Density of actual data coverage behind heat-flow contours is not known.

- Maps not corrected to remove shallow, cold features above hotter, deeper signals.

- Underlying well bottom-hole temperature data is calculated using estimated thermal conductivity and $<75 \%$ of data are equilibrated measurements.
}

E Assumed from studies of analogous geothermal settings, or extrapolated from studies of nearby areas. 


\section{ATTRIBUTE: VOLUME}

\section{Attribute Character Grade}

The volume character grade captures the size of the connected fluid resource based on assessments of reservoir thickness and area. Geothermal systems may contain several distinct reservoirs that are isolated laterally and/or vertically. The reservoir is preferably estimated using a comprehensive numerical and conceptual model because 3-D reservoir modeling is likely to best approximate heterogeneity of the geothermal system. However, such models require several wells with long-term flow test data that can be used to constrain the volume. Therefore, area $\times$ thickness is often used in early phases of exploration to represent a geothermal reservoir, but it is understood to be an oversimplification of the actual reservoir geometry. Guidance for combining area and thickness into a volume measurement only applies if a conceptual or numerical model is not yet available.

The ranges listed in Table 7 are based on the size distribution of identified geothermal resources from the USGS 2008 assessment (Williams et al. 2008).

Table 7. Character Grade for Volume Attribute

\begin{tabular}{ccc} 
Grade & Value Range $\left(\mathrm{km}^{3)}\right.$ & Description \\
A & $>10$ & Very large \\
\hline B & $>5-10$ & Large \\
\hline C & $>2.5-5$ & Moderate \\
\hline D & $>0.5-2.5$ & Small \\
\hline E & $\leq 0.5$ & Very small \\
\hline
\end{tabular}

As shown in Table 7, a resource with a very large volume $\left(>10 \mathrm{~km}^{3}\right)$ is assigned a volume character grade of $A$, whereas a resource with a very small volume $\left(\leq 0.5 \mathrm{~km}^{3}\right)$ would be assigned a volume character grade of $\mathrm{E}$.

To report the volume character grade, select the grade that corresponds to the modeled reservoir volume-or, in early stages of exploration, the volume calculated as the product of the most likely measurements of area and thickness. When reporting volume, consider the conceptual model, activities performed, and quality of the data collected. Often, several activities and data sources can be used to estimate areal extent and thickness estimates; greater confidence in estimates is obtained when independent observations yield similar results.

For further guidance on choosing the most likely, upper, and lower bounds of volume measurements, refer to the section Selecting Estimates of Reservoir Volume in the Resource Size Assessment Tool.

Further details on selecting activity and execution index values are described in the following activity index and execution index sub-sections of Volume Grade.

\section{Activity Index}

The volume activity index represents the ability of exploration activities to represent the volumetric size of a geothermal resource. A geothermal reservoir is defined by the extent of useable heat, which depends on the permeability distribution of the hydrothermal system, or the extent of reservoir 
stimulation in an enhanced geothermal system. As a result, volume is a calculated attribute. This concept is in contrast to oil and ore, where volume is a directly measurable physical entity. This Protocol outlines activities that (indirectly) measure thickness and area components that are used to make volume calculations.

The order of activities listed in Tables 8-1 and 8-2 represents the likelihood that the activities will accurately estimate the areal extent and thickness of the geothermal system. Whether the reported volume grade is based on a reservoir model, or the simpler area $x$ thickness calculation, report an activity index for both area and thickness independently. The lower of the two indices will be used to represent the overall activity index for volume. In practice, all available data sets should be used to develop an internally consistent conceptual model of the resource; this will result in the best estimates of reservoir volume.

Table 8-1. Volume Sub-Attribute AREA, Activity Index

\begin{tabular}{|c|c|c|}
\hline Index & Activity (for Area) & See Related Execution Index \\
\hline A & $\begin{array}{l}\text { Flow testing: multiple wells } \\
\text { with reservoir modeling }\end{array}$ & Table 6-1 \\
\hline B & $\begin{array}{l}\text { Subsurface temperature } \\
\text { probe: multiple wells }\end{array}$ & Table 6-2 \\
\hline C & TEM, MT & Tables 6-3, 6-4 \\
\hline D & $\begin{array}{l}\text { Core stratigraphy, magnetic } \\
\text { surveys }\end{array}$ & Tables 6-5, 6-6 \\
\hline E & $\begin{array}{l}\text { Field mapping/surveys of } \\
\text { surface manifestations, } \\
\text { distribution of hydrothermal } \\
\text { alteration, and bounding } \\
\text { geologic structures }\end{array}$ & Table 6-9 \\
\hline Index & Activity (for Thickness) & See Related Execution Index \\
\hline A & Flow testing: multiple wells & Table 6-1 \\
\hline B & $\begin{array}{l}\text { Subsurface temperature } \\
\text { probe: multiple wells }\end{array}$ & Table 6-2 \\
\hline C & MT & Table 6-4 \\
\hline D & Gravity surveys, TEM, seismic & Tables 6-3, 6-7, 6-8 \\
\hline E & $\begin{array}{l}\text { Field mapping (stratigraphy) / } \\
\text { surveys of surface } \\
\text { manifestations, distribution of } \\
\text { hydrothermal alteration, and } \\
\text { bounding geologic structures }\end{array}$ & Table 6-9 \\
\hline
\end{tabular}

If there is scientific reason to report other than as prescribed, it is important to note this discrepancy and the justification when reporting. 
Refer to the Resource Size Assessment Tool for further guidance in selecting the activity index value that corresponds to the most likely, upper, and lower bounds of measurements for area and thickness.

\section{Execution Index}

For each activity used to measure volume, an execution index reflects how the data were verified relative to best practices. The index quantitatively indicates relative error and uncertainty in volumerelated exploration activities.

The tables below list the primary types of activity used to measure volume. There are two important factors to keep in mind:

- These tables are not an exhaustive list of all possible analytical methods that could be used to estimate volume. Those who have used (or wish to use) an analytical method or technique not listed here should contact the DOE GTO for guidance to create an appropriate execution index for reporting.

- The criteria in these tables are not meant to be guidelines to perform these exploration activities and are not an exhaustive list of all quality controls that must be performed. It is assumed that all analyses are performed by an experienced professional. These tables are for reporting purposes only.

For the selected "most representative" activity used to report volume grade, select the execution index that best corresponds to the quality of information obtained.

Example 1: If volume was determined from flow testing and subsequent reservoir modeling, use Table 8-1 to determine the appropriate execution index (i.e., indicate how well the probe readings were done).

Example 2: If information obtained by a contractor performing the flow test measurements can be shown to meet all the criteria in $A$, report the execution index value as $A$. 
Table 9-1. Execution Index: Flow Tests

Corresponds to sub-attribute Thickness, activity index: A (Tables 8-1) and sub-attribute Area activity index: A (Tables 8-2)

\begin{tabular}{|c|c|}
\hline Index & Execution Details for Flow Tests \\
\hline A & $\begin{array}{l}\text { - Flow tests are completed in full-size large diameter wells. } \\
\text { - Tests performed in multiple wells (more than two). } \\
\text { - Tests include, but are not limited to, pressure buildup and falloff tests, } \\
\text { interference tests, and tracer tests. } \\
\text { - Results correlated with temperature and pressure logs at all wells. } \\
\text { - Test results are interpreted with numerical modeling. }\end{array}$ \\
\hline B & $\begin{array}{l}\text { - Flow tests are completed in small-diameter production wells. } \\
\text { - Tests performed in multiple wells (two or more). } \\
\text { - Multiple test types are performed (e.g., pressure buildup and falloff } \\
\text { tests, interference tests, and tracer tests), but not at all wells. } \\
\text { - Results correlated with temperature and pressure logs at some, but not } \\
\text { all, test wells. } \\
\text { - Test results are interpreted with rudimentary numerical modeling (i.e., } \\
\text { more assumptions of standard values than measured data inputs). }\end{array}$ \\
\hline C & $\begin{array}{l}\text { - Results taken from previous third-party studies of the area (either } \\
\text { literature or contractors) with little or limited information on survey } \\
\text { methods, replication, or error. }\end{array}$ \\
\hline D & $\begin{array}{l}\text { - Flow tests are completed in slim holes. } \\
\text { - Tests performed in only one well. } \\
\text { - Only one type of test is performed (e.g., pressure buildup and falloff } \\
\text { tests, interference tests). } \\
\text { - Results not correlated with temperature and pressure logs. } \\
\text { - No numerical modeling of results. }\end{array}$ \\
\hline $\mathbf{E}$ & $\begin{array}{l}\text { - Assumed from studies of analogous geothermal settings or } \\
\text { extrapolated from studies of nearby areas. }\end{array}$ \\
\hline
\end{tabular}


Table 9-2. Execution Index: Subsurface Temperature Probe

Corresponds to sub-attribute Thickness, activity index: A (Tables 8-1) and sub-attribute Area activity index: A (Tables 8-2)

Index

A

\section{Execution Details for Subsurface Temperature Probe}

- Multiple distributed deep wells include both productive and unproductive locations, defining the entire field boundary.

- Temperature gradients for productive wells show near-isothermal temperature profile within the reservoir interval.

- Cuttings and/or geophysics confirms that bottom of well intersects the reservoir (i.e., downhole alteration mineralogy consistent with reading).

B

- Multiple distributed deep wells include some unproductive wells along only a portion of the field extent.

- Temperature gradients for wells within field mostly show nearisothermal temperature profiles.

- Some cuttings and/or geophysics have not confirmed measurement within the reservoir (i.e., downhole alteration mineralogy not consistent with readings).

C

- Results taken from previous third-party studies of the area (either literature or contractors) with little or limited information on survey methods, replication, or error.

D

- Wells are drilled in limited locations.

- No temperature gradients show isothermal temperature profiles-or the base of the isothermal section has not been encountered.

- Cuttings and/or geophysics have not confirmed measurement within the reservoir.

E

- Assumed from studies of analogous geothermal settings or extrapolated from studies of nearby areas. 
Table 9-3. Execution Index: Transient Electro Magnetic (TEM)

Corresponds to sub-attribute Area, activity index: C (Table 8-1) and sub-attribute Thickness, activity index: D (Table 8-2)

Index Execution Details for TEM (Transient Electro Magnetic)

A - Depth of survey is adjusted to the resistivity of the area (e.g., few hundred meters in low resistivity).

- Current is applied to the transmitter loop for a sufficient time.

- Current is shut off abruptly.

- Measurement windows/sampling "gates" are high resolution to capture detailed changes in signal amplitude.

- Conducted in area of minor external noise and interference (or survey includes a quiet remote station to remove noise signal).

- Loop size is more than adequate.

- Spacing between stations is adequately close to capture variability in features.

- Areal extent of survey shows all field boundaries.

- 2-D and 3-D inversions are performed.

B - Depth of survey is appropriate to resistivity of area (few hundred meters in low resistivity, up to $1 \mathrm{~km}$ in high resistivity).

- Current is applied to the transmitter loop for a sufficient time.

- Current is shut off abruptly.

- Measurement windows/sampling "gates" are adequate to capture relevant changes in signal amplitude.

- Conducted in area of some minor external noise and interference (or survey includes a quiet remote station that does not fully remove noise signal).

- Loop size is appropriate for area.

- Spacing between stations is adequately close to capture variability in features in some areas but not all.

- Areal extent of survey shows some field boundaries but not all.

- 2-D and/or 3-D inversions are performed.

C - Results taken from previous third-party studies of the area (either literature or contractors) with little or limited information on survey methods, replication, or error.

D - Effective exploration depth is not appropriately adjusted to resistivity of area (e.g., $1 \mathrm{~km}$ in low resistivity).

- Current is not applied to the transmitter loop for a sufficient time.

- Current is not shut off abruptly.

- Measurement windows/sampling "gates" are too wide to capture relevant changes in signal amplitude.

- Conducted in area of significant external noise and interference (and/or survey does not include a quiet remote station).

- Loop size is too small for area.

- Spacing between stations does not adequately capture variability in features in any given area.

- Areal extent of survey does not indicate field boundaries.

- 2-D inversions are performed.

E Assumed from studies of analogous geothermal settings or extrapolated from studies of nearby areas. 
Table 9-4. Execution Index: Magnetotelluric (MT)

Corresponds to sub-attribute Area, activity index: C (Table 8-1) and sub-attribute Thickness, activity index: C(Table 8-2)

Index

A

\section{Execution Details for MT (Magnetotelluric)}

- Local resistivity anomalies are known and have been used to manually correct the telluric shift (e.g., through inversion of TEM results).

- Minimal incidents of signal noise (such as cultural interference), or survey includes a quiet remote station to remove noise signal.

- Measurements are taken over several hours at each site.

- Frequency of the signal is appropriate to the depth being probed (e.g., $0.00001-10 \mathrm{~Hz}$ for deep crustal investigations and $10-1000 \mathrm{~Hz}$ for upper crust features).

- Spacing between stations is adequately close to capture variability in features.

- Areal extent of survey shows all field boundaries.

- 2-D and 3-D inversions are performed.

B

- Local resistivity anomalies are relatively well known and have been used to manually correct the telluric shift.

- Some incidents of signal noise (such as cultural interference) or survey includes a quiet remote station that does not fully remove noise signal.

- Measurements are taken over several hours at each site.

- Frequency of the signal is appropriate to the depth being probed.

- Spacing between stations is adequately close to capture variability in features.

- Areal extent of survey shows all field boundaries.

- 2-D and/or 3-D inversions are performed.

C

- Results taken from previous third-party studies of the area (either literature or contractors) with little or limited information on survey methods, replication, or error.

D

- Local resistivity anomalies are not well known and corrections to the telluric shift are assumed.

- Some significant incidents of signal noise and/or survey does not include a quiet remote station.

- Measurements are taken for the minimum time possible at each site.

- Frequency of the signal not fully appropriate to the depth being probed.

- Spacing between stations does not adequately capture variability in features in any given area.

- Areal extent of survey does not indicate field boundaries.

- 2-D inversions are performed. studies of nearby areas. 
Table 9-5. Execution Index: Core Stratigraphy

Corresponds to sub-attribute Area, activity index: D (Table 8-1)

\section{Index Execution Details for Core Stratigraphy}

A - Well cuttings and core have been taken over a wide areal extent to map relationships between rock units, hydrothermal alteration, and geologic structures.

- Stratigraphic sequences and hydrothermal alteration zones correlated across multiple deep wells.

- Surface geologic mapping has been completed in sufficient detail to correspond with structures at depth.

- Includes combination of visual inspection by binocular microscope, petrographic thin sections, X-ray diffraction (XRD) clay analysis (if applicable), and fluid-inclusion measurements.

B - Well cuttings and core are complete enough to confirm major relationships between rock units, hydrothermal alteration, and geologic structures.

- Stratigraphic sequences and hydrothermal alteration zones correlated across multiple deep wells.

- Surface geologic mapping has been completed in sufficient detail to estimate stratigraphy and structures at depth.

- Includes combination of visual inspection by binocular microscope, petrographic thin sections, and XRD clay analysis (if applicable).

C - Results taken from previous third-party studies of the area (either literature or contractors) with little or limited information on survey methods, replication, or error.

D - Well cuttings and core are not complete enough to confirm major relationships between rock complexes, hydrothermal alteration, and geologic structures.

- Stratigraphic sequences and hydrothermal alteration zones not followed across multiple deep wells.

- Surface geologic mapping has not been completed in sufficient detail to estimate stratigraphy and structures at depth.

- Includes combination of visual inspection by binocular microscope and petrographic thin sections.

E Assumed from studies of analogous geothermal settings or extrapolated from studies of nearby areas. 
Table 9-6. Execution Index: Magnetic Surveys

Corresponds to sub-attribute Area, activity index: D (Table 8-1)

Index

A

\section{Execution Details for Magnetic Surveys}

- On-the-ground measurements are spaced in parallel profiles or grids at high resolution for the features under investigation.

- Survey lines are tailored in detail to follow geologic strike.

- Initial clear magnetic signature is seen, and alteration events are known/well studied.

- Aeromagnetic surveys of large-scale features are available to compliment on-the-ground results, particularly in sedimentary basins.

- Location of magnetic signatures can be corroborated with other methods (e.g., gravity).

B

- On-the-ground measurements are spaced in parallel profiles or grids at an appropriate resolution for the features under investigation.

- Survey lines follow geologic strike, where known.

- Location of magnetic signatures cannot be corroborated with other methods, but contains GPS corrections to within 5 meters.

- Data gaps due to land access issues/steep terrain were overcome by aeromagnetic surveys.

C

- Results taken from previous third-party studies of the area (either literature or contractors) with little or limited information on survey methods, replication, or error.

D

- Land access issues/steep terrain only allow intermittent sampling.

- Measurements of local structures are inferred from lower-resolution aeromagnetic surveys.

- Surface structure extrapolated to infer deeper intrusions, without an aeromagnetic survey.

- Location of magnetic signatures cannot be corroborated with other methods, and conditions force GPS correction accuracy $>5$ meters.

- Survey lines are oriented without consideration of geologic strike.

- Assumed from studies of analogous geothermal settings or extrapolated from studies of nearby areas. 
Table 9-7. Execution Index: Gravity Surveys

Corresponds to sub-attribute Thickness, activity index: D (Table 8-2)

Index

A

\section{Execution Details for Gravity Surveys}

- The line direction is positioned perpendicular to the dominant geologic strike.

- Intervals are spaced finely enough to individually characterize all anticipated anomalies.

- Precise measurements of altitude, rock mass, and local topography used to inform corrections.

- Appropriate Bouger, free air, and/or terrain corrections performed. Deep wells exist that can be used to constrain gravity model.

B

- The line direction is positioned perpendicular to the dominant geologic strike.

- Intervals are spaced finely enough to individually characterize all anticipated anomalies.

- Precise measurements of altitude and rock mass are used to inform corrections. Local topographic maps or digital elevation models are used, but are of unknown quality/not recently updated.

C

- Results taken from previous third-party studies of the area (either literature or contractors) with little or limited information on survey methods, replication, or error.

D

- Use of imprecise values for altitude, rock mass, and/or topography, thereby estimating free-air, Bouguer, and/or terrain corrections.

- Intervals spaced to capture some (but not all) anomalies.

- Sampling includes only one measurement per anomaly.

- Line direction is not fully perpendicular to dominant geologic strike, strike is not known, and/or stratigraphy and structure of the region is not well constrained.

- Assumed from studies of analogous geothermal settings or extrapolated from studies of nearby areas. 
Table 9-8. Execution Index: Active Seismic Reflection

Corresponds to sub-attribute Thickness, activity index: D (Table 8-2)

Index

A

\section{Execution Details for Active Seismic Reflection}

- Used in areas dominated by, or overlain by, sedimentary formations.

- Choice of seismic source allows for signal penetration at or below the estimated reservoir depth.

- Geophones are appropriately grounded and secured.

- Survey array provides high-resolution coverage (for both depth and areal extent) in areas of desired feature discovery.

B

- Choice of seismic source allows for signal penetration to a depth that appears to show the top of the reservoir/clay cap.

- Geophones are appropriately grounded and secured, although in loose soil in some areas.

- Survey array adequately corresponds to desired feature discovery.

- Results taken from previous third-party studies of the area (either literature or contractors) with little or limited information on survey methods, replication, or error.

D

- Used in areas not dominated by sedimentary formations (e.g., fractured crystalline rock types), resulting in high uncertainty in interpretation.

- Choice of seismic source does not allow for signal penetration to the estimated reservoir top depth. Geophones have not been checked for security or grounding or are within very loose soil.

- Survey array is sparse, with little correspondence to desired feature discovery. from studies of nearby areas. 
Table 9-9. Execution Index: Field Mapping

Corresponds to sub-attribute Area, activity index: $E$ (Table 8-1) and sub-attribute Thickness, activity index: $E$ (Table 8-2)

Index

A

\section{Execution Details for Field Mapping}

- Field studies include comprehensive mapping of structures, stratigraphy, and location of surface manifestations and hydrothermal alteration.

- Mapping is completed at a fine level of detail within the exploration area.

- Mapped characteristics and zones of identified hydrothermal alteration are supported by remote sensing data (such as satellite imagery, air photos, or LiDAR).

B

- Field studies include mapping of stratigraphy, fault geometry, and location of surface manifestations.

- Mapping is completed at a moderate level of detail within the exploration area (e.g., major faults).

- Mapped characteristics and zones of identified hydrothermal alteration are partially supported by remote sensing data (such as satellite imagery, air photos, or LiDAR).

C

- Results taken from previous third-party studies of the area (either literature or contractors) with little or limited information on survey methods, replication, or error.

D

- Field studies include cursory stratigraphic and fault geometry review, adequate location of surface manifestations.

- Mapping is completed at a moderate level of detail within the exploration area (e.g., regional features only).

- Mapped characteristics and zones of identified hydrothermal alteration are not supported/verified by the use of remote sensing data (such as satellite imagery, air photos, or LiDAR).

$\mathbf{E}$

- Assumed from studies of analogous geothermal settings or extrapolated from studies of nearby areas.

Constraints to surficial mapping of geologic and thermal features used to identify the extent of the geothermal system include: 1) the level of detail of the mapping itself; and 2) other externalities such as the nature of the exposures (bedrock versus alluvium cover), amount of vegetation, and amount of topographic relief. 


\section{ATTRIBUTE: PERMEABILITY}

\section{Attribute Character Grade}

Unlike temperature and volume, a classification system does not currently exist to characterize a geothermal reservoir's permeability. Geothermal reservoirs exhibit two types of permeability: matrix permeability and fracture permeability, which tend to differ by orders of magnitude. Fluid flow predominantly occurs in the fracture network for most hydrothermal systems, but the bulk of the fluid is typically contained in matrix pores. Although "representative" permeability values for geothermal wells are often reported as $K_{h}$ (or permeability-thickness) values, this singular value does not capture the heterogeneous nature of the system.

Due to the difficulty in defining quantitative values for permeability applicable to all geothermal resources, GeoRePORT approximates differences in permeability through five qualitative sub-attributes, as shown in Table 10-1 and described in further detail in Tables 10-2 to 10-6.

Table 10-1. Permeability Sub-Attributes

\begin{tabular}{l} 
Sub-Attribute \\
Fault/fracture orientation \\
\hline Effective fault/fracture aperture \\
\hline Mineralization \\
\hline Fracture spacing \\
\hline Fracture roughness
\end{tabular}

For each of the sub-attributes, report the grade that most represents that resource's behavior.

Example: As shown in Table 10-5, a resource with a close-spaced fracture distribution would be assigned a fracture distribution grade of $A$, whereas a resource with alteration filling all openings (Table 10-4) would be assigned a mineralization grade of $\mathrm{E}$.

Although the grades for fracture orientation, effective aperture, mineralization, and fracture distribution are independent, report the grade that best represents the largest influence over the permeability of the system.

Example: If the mineralization grade and fracture aperture grade are $A$, and the feature distribution grade is $B$, report the fluid permeability grade $B$ if the fracture distribution is notably limiting the overall resource permeability.

When reporting Permeability Grade, consider the conceptual model, activities performed, and quality of the data collected. Often, several activities and data sources can be used to estimate permeability; greater confidence in estimates is obtained when independent observations yield similar results. 
Table 10-2. Permeability Sub-Attribute: Fault/Fracture Orientation

\begin{tabular}{|c|c|c|}
\hline Grade & & Fault and Fracture Orientation \\
\hline A & $\begin{array}{l}\text { Very } \\
\text { high }\end{array}$ & Optimally oriented for dilation and sheer, with abundant intersections \\
\hline B & High & Optimally oriented for dilation and shear, with some intersections \\
\hline C & Medium & Intersecting features, but not optimally oriented for dilation and shear \\
\hline D & Low & Not optimally oriented for dilation and shear, with few intersections \\
\hline E & $\begin{array}{l}\text { Very } \\
\text { low }\end{array}$ & $\begin{array}{l}\text { Poorly oriented for dilation and shear, and little to no intersection of } \\
\text { features }\end{array}$ \\
\hline
\end{tabular}

Table 10-3. Permeability Sub-Attribute: Effective Fault/Fracture Aperture

\begin{tabular}{ccl} 
Grade & Description & \multicolumn{1}{c}{ Effective Aperture } \\
A & Very high & Very large $(>25 \mathrm{~mm})$ \\
\hline B & High & Large $(>10-25 \mathrm{~mm})$ \\
\hline C & Medium & Moderate $(>5-10 \mathrm{~mm})$ \\
\hline D & Low & Small $(>1-5 \mathrm{~mm})$ \\
\hline E & Very Low & Very small $(\leq 1 \mathrm{~mm})$ \\
\hline
\end{tabular}

Table 10-4. Permeability Sub-Attribute: Mineralization

\begin{tabular}{|c|c|c|}
\hline Grade & Description & Mineralization \\
\hline A & Very low & $\begin{array}{l}\text { Minimal evidence of alteration, or fractures } \\
\text { are calcite or quartz filled (multiple episodes } \\
\text { of reopening). No clay alteration/fracture fill. }\end{array}$ \\
\hline B & Low & $\begin{array}{l}\text { Precipitation in fractures or vesicles is mostly } \\
\text { calcite or quartz, but exhibits some clay fill (< } \\
10 \%)\end{array}$ \\
\hline C & Medium & $\begin{array}{l}\text { Clay mineral precipitation evident }(<33 \%) \text {, } \\
\text { but not pervasive }\end{array}$ \\
\hline D & High & $\begin{array}{l}\text { Areas of significant clay alteration ( }<\text { or }= \\
50 \% \text { of fractures filled with clay) }\end{array}$ \\
\hline $\mathbf{E}$ & Very high & $\begin{array}{l}\text { Significant clay mineralization (> 50\% of } \\
\text { fractures) }\end{array}$ \\
\hline
\end{tabular}


Table 10-5. Permeability Sub-Attribute: Fracture Spacing

\begin{tabular}{ccl} 
Grade & Description & \multicolumn{1}{c}{ Fracture Spacing } \\
A & Very high & Fractures closely spaced $(<0.5 \mathrm{~m})$ \\
\hline B & High & Fractures moderately spaced $(0.5-5 \mathrm{~m})$ \\
\hline C & Medium & $\begin{array}{l}\text { Fractures or faults inconsistently } \\
\text { spaced }(5-10 \mathrm{~m})\end{array}$ \\
\hline D & Low & $\begin{array}{l}\text { Singular or few dominant faults }(10-50 \\
\mathrm{m})- \text { connected }\end{array}$ \\
\hline E & Very low & $\begin{array}{l}\text { Singular or few dominant faults }(>50 \\
\mathrm{m})- \text { no interaction }\end{array}$ \\
\hline
\end{tabular}

Table 10-6. Permeability Sub-Attribute: Fracture Roughness²

\begin{tabular}{ccl} 
Grade & Description & $\begin{array}{l}\text { Fracture Roughness (root mean } \\
\text { square, rms) }\end{array}$ \\
A & Very high & $\begin{array}{l}\text { Significant fracture roughness ( } \\
10 \mathrm{~cm} \mathrm{rms})\end{array}$ \\
\hline B & High & $\begin{array}{l}\text { High fracture roughness }(5-10 \mathrm{~cm} \\
\mathrm{rms})\end{array}$ \\
\hline C & Medium & Moderate roughness $(2-5 \mathrm{~cm} \mathrm{rms})$ \\
\hline D & Low & Limited roughness $(1-2 \mathrm{~cm} \mathrm{rms})$ \\
\hline E & Very Low & $\begin{array}{l}\text { Smooth fracture surfaces }(<1 \mathrm{~cm} \\
\mathrm{rms})\end{array}$ \\
\hline
\end{tabular}

Another aspect of fracture permeability that is important (and hard to quantify) is the distribution of fracture lengths. Core samples and image logs provide constraints on the strike, dip, frequency, mineralization, and aperture of fractures, but not fracture length. Longer fractures potentially favor enhanced permeability because they increase the possibility for intersecting other features; they may also serve as fast path conduits for fluid flow within the reservoir. However, due to this uncertainty in measuring representative fracture length and length distribution, this feature is not included in the above index.

Refer to the activity index and execution index sub-sections of this Permeability Grade section for more details on activities to evaluate permeability characteristics. For further guidance on choosing the most likely, upper, and lower bounds on estimates of permeability, refer to the Resource Size Assessment Tool.

${ }^{2}$ Root mean square roughness is the quadratic mean of the vertical deviations of the roughness profile:
surface roughness to be characterized by a single value: the larger the deviations, the greater the surface roughness. 


\section{Activity Index}

Permeability cannot be directly measured. The activity index (Table 11) lists activities ordered by their ability to represent reservoir permeability. For example, the transmissivity of a reservoir can be directly determined through flow tests (Index A), which can be used to constrain the overall permeability.

Table 11. Activity Index for Permeability Attribute

Index

A

A

A

\begin{tabular}{clc}
\multicolumn{1}{c}{ • Tracer tests } & \\
\hline B & $\begin{array}{l}\text { Lithologic cores (and laboratory measurements) } \\
\text { and formation microimaging-BHTV or acoustic } \\
\text { reflectivity }\end{array}$ & Table 12-4, 12-5 \\
\hline C & Fault dilation analysis & Table 12-6 \\
\hline D & $\begin{array}{l}\text { Structural field mapping; distribution of thermal } \\
\text { features }\end{array}$ & Table 12-7 \\
\hline E & $\begin{array}{l}\text { Vertical seismic profiling (VSP) and reflection } \\
\text { seismic; passive seismic }\end{array}$ & Table 12-8, 12-9 \\
\hline
\end{tabular}

Decisions on the most appropriate activity index to report should be done within the context of the conceptual model. This integrated approach, which incorporates all types of permeability data, is likely to better capture the heterogeneity in the reservoir. Although the indices for Fault/Fracture Orientation, Mineralization, Effective Aperture, Fracture Spacing, and Surface Roughness are independent, report the activity index for the grade that best represents the largest influence over the permeability of the system.

Example: If the activity index for Mineralization and Fracture Aperture is $A$ and for Feature Distribution is $B$, but the fracture distribution is the limiting factor to access overall permeability, report the activity index as $B$.

If there is scientific reason to report other than as prescribed, it is important to note this discrepancy and the justification when reporting.

Refer to the Resource Size Assessment Tool for further guidance in selecting the activity index value that corresponds to the most likely, upper, and lower bounds of measurements for permeability.

\section{Execution Index}

For each activity used to estimate permeability, an execution index reflects how the data were verified relative to best practices. The index quantitatively indicates relative error and uncertainty in permeability-related exploration activities. 
The tables below list the primary types of activity used to estimate permeability. There are two important factors to keep in mind:

- These tables are not an exhaustive list of all possible analytical methods that could be used to estimate permeability. Those who have used (or wish to use) an analytical method or technique not listed here should contact the DOE GTO for guidance to create an appropriate execution index for reporting.

- The criteria in these tables are not meant to be guidelines to perform these exploration activities and are not an exhaustive list of all quality controls that must be performed. It is assumed that all analyses are performed by an experienced professional. These tables are for reporting purposes only.

For the selected "most representative" activity used to report permeability grade, select the execution index that best corresponds to the quality of information obtained.

Example: If permeability was inferred from flow testing, use Table 12-1 to determine the appropriate execution index (i.e., indicate how well the probe readings were done). If information obtained by a contractor performing the flow test measurements can be shown to meet all the criteria in $A$, then report the index value as $A$. 
Table 12-1. Execution Index: Flow and/or Injection Tests

Corresponds to activity index: $A$ (Table 11)

\section{Index Execution Details for Flow and/or Injection Tests}

A - Flow or injection tests are completed in full-size large diameter wells.

- Tests performed in multiple wells (more than two).

- Tests include, but are not limited to pressure buildup and falloff tests, interference tests, step-rate tests, and tracer tests.

- Test duration of $>4$ weeks.

- Results correlated with temperature and pressure logs at all wells.

B - Flow tests are completed in small-diameter production wells.

- Tests performed in multiple wells (two or more).

- Multiple test types are performed (e.g., pressure buildup and falloff tests, interference tests, steprate tests, and tracer tests), but not at all wells.

- Test duration of 1 to 4 weeks.

- Results correlated with temperature and pressure logs at some, but not all, test wells.

C - Results taken from previous third-party studies of the area (either literature or contractors) with little or limited information on survey methods, replication, or error.

D $\quad$ Flow tests are completed in slim holes.

- Tests performed in only one well.

- Only one type of test is performed (e.g., pressure buildup and falloff tests, step-rate tests, interference tests).

- Test duration of 1 week or less.

- Results not correlated with temperature and pressure logs.

E Assumed from studies of analogous geothermal settings or extrapolated from studies of nearby areas. 
Table 12-2. Execution Index: Tracer Test

Corresponds to activity index: A (Table 11)

\section{Index Execution Details for Tracer Test}

A - Tracer is appropriate for the reservoir temperature and type (liquid or steam dominated).

- Measured at regular, frequent intervals.

- Measured directly and continuously online.

- No other wells in the flow path between the injection and monitoring wells.

- Liquid- and vapor-phase tracers are injected with precisely metered rates.

- Tracer is conservative and not thermally sensitive.

- Pulsed Injection Strategy utilized (tracer is injected as aliquots at a constant rate for a period of time sufficient for the downstream concentration to reach a steady-state equilibrium value).

- Small, known quantity of fluid is injected after the tracer.

B - Tracer is appropriate for the reservoir temperature or type (liquid- or steam-dominated).

- Not measured in real time.

- Other injection wells are nearby, but not directly in, the flow path between the tracer's injection and monitoring wells.

- Liquid- and vapor-phase tracers are injected with precisely metered rates.

- Tracer is conservative but exhibits some thermal sensitivity that is known.

- Pulsed Injection Strategy utilized (tracer is injected as aliquots, but downstream concentration does not reach a steady-state equilibrium value).

- Moderately large, but known, quantity of fluid is injected after the tracer.

C $\quad$ Results taken from previous third-party studies of the area (either literature or contractors) with little or limited information on survey methods, replication, or error.

D - Tracer is not appropriate for either the reservoir temperature or type (liquid- or steam-dominated).

- Other injection wells are operating in the flow path between the tracer's injection and monitoring wells.

- Liquid- and vapor-phase tracers are injected with some error (metered rates are not consistent).

- Tracer is not conservative and is thermally sensitive.

- Slug Injection Strategy: utilized (tracer is injected as a single slug).

- Moderately large unmeasured quantity of fluid is injected after the tracer.

E Assumed from studies of analogous geothermal settings or extrapolated from studies of nearby areas. 
Table 12-3. Execution Index: Temperature Logs

Corresponds to activity index: $B$ (Table 11)

Index Execution Details for Temperature Logs

A - Log clearly shows anomalies or inflections after well flowing or cold-water injection.

- Corresponding core available that demonstrates the presence of alteration mineral morphologies indicative of open fractures (e.g., bladed calcite, euhedral quartz crystals).

- Log was run under flowing conditions.

- DTS and/or PTS logs clearly identify depths and magnitude of fluid entries in the well bore.

- Rate of wireline temperature log is slow enough to resolve all fluid entry depths with other measurements.

- Identified flow zones correlate with drilling fluid loss zones; fracture zones identified in image logs and core.

B - Log clearly shows anomalies or inflections after well flowing or cold-water injection.

- Drill cuttings available that indicate the presence of alteration mineral morphologies indicative of open fractures (e.g., bladed calcite, euhedral quartz crystals).

- Log was run under injecting conditions.

- DTS and/or PTS logs identify some, but not all, depths and magnitude of fluid entries in the well bore.

- Rate of wireline temperature log is slow enough to resolve some fluid entry depths with other measurements.

C - Results taken from previous third-party studies of the area (either literature or contractors) with little or limited information on survey methods, replication, or error.

D - Log does not clearly show anomalies or inflections even after well flowing or cold-water injection.

- Neither corresponding drill cuttings nor core available.

- Log was run under static conditions.

- DTS and/or PTS logs identify few depths and magnitude of fluid entries in the well bore.

- Rate of wireline temperature log is too fast to resolve fluid entry depths with other measurements.

E - Assumed from studies of analogous geothermal settings or extrapolated from studies of nearby areas. 
Table 12-4. Execution Index: Lithologic Cores

Corresponds to activity index: $B$ (Table 11)

\section{Index Execution Details for Lithologic Cores}

A - Core oriented with an image log.

- Follows consistent labeling, record keeping, and description methods.

- Hydrothermal alteration minerals examined in thin section.

- Frequency of faulting and fracture orientation measured.

- Stratigraphic sequences well identified when core is recovered.

- Good recovery over cored interval.

B - Core oriented by visual inspection.

- Follows consistent labeling, record keeping, and description methods.

- Hydrothermal alteration minerals examined in thin section.

- Frequency of faulting and fracture orientation measured.

- Stratigraphic sequences mostly identified when core is recovered.

C - Results taken from previous third-party studies of the area (either literature or contractors) with little or limited information on survey methods, replication, or error.

D - Core received from previous studies and/or core is not oriented.

- Evidence of inconsistent labeling, record keeping, and description methods.

- Spot examinations of hydrothermal alteration minerals in thin section.

- Frequency of faulting and fracture direction not noted in core log descriptions.

- No cohesive map correlating stratigraphic sequences from regional cores.

E - Assumed from studies of analogous geothermal settings or extrapolated from studies of nearby areas. 
Table 12-5. Execution Index: Formation Micro Imager Logs

Corresponds to activity index: $C$ (Table 11)

Index Execution Details for Formation Micro Imager Logs

A - Combined with other geophysical wireline measurements (e.g., azimuthal resistivity imager, or induction imager, gamma log) to identify drilling-induced fractures and borehole breakouts.

- Available for significant thickness/depth of reservoir (i.e., able to identify heterogeneity).

- Evaluated for fractures, faults, stress direction, and lithology.

- Corresponds to other lithology cores and subsurface geology.

- Imaging run with sufficient injection flow to keep instrument below maximum operating temperature.

- Log depth is properly registered (either from the Kelly bushing [KB] or the wellhead).

B - Combined with minimal geophysical wireline measurements (e.g., temperature, pressure).

- Available for significant proportion of reservoir.

- Evaluated for fractures, faults, stress direction, and lithology.

- Minimally corresponds to other lithology cores and subsurface geology (e.g., cannot fully trace faults).

- Imaging run with intermittent injection flow to regulate instrument temperature.

- Log depth is properly registered (either from the KB or the wellhead).

C - Results taken from previous third-party studies of the area (either literature or contractors) with little or limited information on survey methods, replication, or error.

D - Combined with no other geophysical wireline measurements (e.g., temperature, pressure).

- Available for small, limited part of the reservoir.

- Evaluated for some, but not all: stress direction, fractures, faults, and lithology.

- Does not correspond to other lithology cores and subsurface geology (e.g., cannot fully trace faults).

- Imaging run without injection flow.

- Log depth is not properly registered.

E Assumed from studies of analogous geothermal settings or extrapolated from studies of nearby areas. 
Table 12-6. Execution Index: Acoustic Reflectivity

Corresponds to activity index: C (Table 11)

\section{Index Execution Details for Acoustic Reflectivity}

A - Acoustic caliper, transit times, and amplitude of televiewer logs are collected at high quality to identify fine fracture permeability.

- Location, strike and dip of fractures, and lithologic contacts can be identified in all logs.

- Entire signal is digitized as waveform.

- Cycle skipping can be constrained to identify fractures (i.e., not due to improper signal, detection level, or gas in the fluid).

- Spacing (1-ft receiver) allows identification of lithologic contacts as sharp deflections.

B - Acoustic caliper, transit times, and amplitude of televiewer logs are collected at sufficiently high quality to identify fracture-driven permeability.

- Location, strike and dip of fractures, and lithologic contacts can be identified in vast majority of logs.

- Entire signal is digitized as waveform.

- Cycle skipping can mostly be constrained to identify fractures (i.e., minor issues due to improper signal, detection level, or gas).

- Spacing (1-ft receiver) allows identification of lithologic contacts as sharp deflections.

C - Results taken from previous third-party studies of the area (either literature or contractors) with little or limited information on survey methods, replication, or error.

D - Acoustic caliper, transit times, and amplitude of televiewer logs are collected at moderate quality, enough to generally characterize fracture-driven permeability.

- Location, strike and dip of fractures, and lithologic contacts can be identified in most logs.

- Entire signal is digitized as waveform.

- Cycle skipping prevents fracture identification (i.e., identifiable issues due to improper signal, detection level, or gas in the fluid).

- Spacing does not allow identification of lithologic contacts as sharp deflections.

E Assumed from studies of analogous geothermal settings or extrapolated from studies of nearby areas. 
Table 12-7. Execution Index: Vertical Seismic Profiling and Reflection Seismic

Corresponds to activity index: $D$ (Table 11)

\section{Index Execution Details for VSP and Reflection Seismic}

A - Results correspond to fracture systems and stratigraphy represented in well logs.

- Interpreted sections identify major seismic reflectors and faults as an overlay to the actual data.

- Cross sections are provided both with and without interpretation.

- 3-D reflection results.

- VSP available with multicomponent surface data.

- Appropriate inversions and corrections to explain amplitude and frequency variation.

- Reliable seismic velocity model is available to permit conversion from time to depth.

B - Results mostly correspond with fracture systems and well log stratigraphy.

- Cross sections are provided both with and without interpretation.

- 3-D reflection results.

- VSP available with multicomponent surface data.

- Most appropriate corrections to explain amplitude and frequency variation (i.e., frequency dependent-amplitude variation with offset and azimuth (AVO-AVA) as a function of azimuth.

- Reliable seismic velocity model is available to permit conversion from time to depth.

C - Results taken from previous third-party studies of the area (either literature or contractors) with little or limited information on survey methods, replication, or error.

D - Results only loosely correspond to fracture systems and well log stratigraphy.

- Cross sections are provided with interpretation only.

- 2-D reflection results.

- VSP not available with multicomponent data.

- No (or limited) corrections to explain amplitude and frequency variation.

- Seismic velocity model is available to permit conversion from time to depth but is of unknown quality.

E - Assumed from studies of analogous geothermal settings or extrapolated from studies of nearby areas. 
Table 12-8. Execution Index: Passive Seismic

Corresponds to activity index: $D$ (Table 11)

\section{Index Execution Details for Passive Seismic}

A - Pumping tests monitoring seismic activity from pressure changes have been completed.

- Extensive knowledge of subsurface seismic velocities: Well-documented historical records of natural seismic activity covering the entire geothermal field OR significant time series and resolution to identify areas of active faults and fissures.

- Post-survey analysis tying fluid flow during injection and/or discharge to seismic emissions.

- High-quality subsurface geophones and detailed gridded monitoring network.

B - Pumping tests have been completed, and seismicity partially monitored (selected areas, or only after evidence of earthquakes).

- Documented historical records of natural seismic activity covering the majority of geothermal field OR time series and resolution to identify areas of active faults and fissures.

- Post-survey analysis limited to evaluating fluid flow during injection and/or discharge in light of seismic emissions.

- Moderate-quality subsurface geophones and/or monitoring network with moderate resolution.

C - Results taken from previous third-party studies of the area (either literature or contractors) with little or limited information on survey methods, replication, or error.

D - Pumping tests have been completed, and seismicity partially monitored (selected areas, or only after evidence of earthquakes).

- Limited historical records of natural seismic activity covering the selected areas of the geothermal field OR seismicity assumed from analog areas.

- No attempt made to tie areas of injection and/or discharge with seismic emissions.

- No or low-quality subsurface geophones and/or minimal monitoring network.

E - Assumed from studies of analogous geothermal settings or extrapolated from studies of nearby areas. 
Table 12-9. Execution Index: Field Mapping - Structures and Thermal

Corresponds to activity index: E (Table 11)

Index Execution Details for Field Mapping-Structures and Thermal Features

A - Analyst interpreting results has $>5$ years field experience interpolating subsurface structure from surficial features.

- Field studies include comprehensive fault kinematics and stress, overlying stratigraphy, and location of surficial manifestations.

- Scale of mapping comprehensively captures entire geothermal field, including pertinent small-scale and regional structures.

- Surface mapping results correlate highly $\left(R^{2}>0.5\right)$ with interpreted structural features identified from remote-sensing techniques (e.g., LiDAR).

B - Analyst interpreting results has 2 to 5 years field experience interpolating subsurface structure from surficial features. Field studies include comprehensive stratigraphy, fault geometry, and location of surficial manifestations, but minimal study of fault kinematics.

- Scale of mapping captures most of geothermal field.

- Surface mapping results show some correlation $\left(0.25<\mathrm{R}^{2}<0.5\right)$ with interpreted structural features identified from remote-sensing techniques (e.g., LiDAR).

C - Results taken from previous third-party studies of the area (either literature or contractors) with little or limited information on survey methods, replication, or error.

D - Analyst has minimal background ( $<2$ years) field experience interpolating subsurface structure from surficial features.

- Field studies include cursory stratigraphic and fault geometry review, adequate location of surficial manifestations, and no fault kinematics.

- Scale of mapping covers only part of geothermal field.

- Surface mapping results shows little or no correlation $\left(R^{2}<0.25\right)$ with interpreted structural features identified from remote-sensing techniques (e.g., LiDAR).

E - Assumed from studies of analogous geothermal settings or extrapolated from studies of nearby areas. 


\section{ATTRIBUTE: FLUID CHEMISTRY}

\section{Attribute Character Grade}

The chemical composition of geothermal fluids determines their reactivity-both with the surrounding rock and the well casing in the subsurface, and with surface equipment such as wellheads and piping. The chemistry of a geothermal system can be an immediate deterrent to further exploration if found to be too caustic or acidic for traditional equipment. This Protocol does not fingerprint all types of geothermal waters; instead, the Fluid Chemistry Grade is focused on the characteristics of geothermal chemistry that may impede or benefit development.

Multiple facets of brine and gas chemistry affect reactivity. For example, low-pH fluids or species such as $\mathrm{HCl}$ can cause corrosion, whereas highly saline fluids can cause mineral precipitation, i.e., "scaling," as shown in Table 13 and described in further detail in Tables 14-1A to 14-6A.

Table 13. Fluid Chemistry Sub-Attributes

\section{Sub-Attribute}

$\mathrm{pH}$

Corrosive gas content $\left(\mathrm{HCl}, \mathrm{SO}_{2}\right.$, and/or $\left.\mathrm{H}_{2} \mathrm{~S}\right)$

Non-condensable gas content

Bicarbonate content

Total dissolved solid content

Silica content

Each reservoir should be assigned a grade for each of the sub-attributes.

Example: A resource with a $\mathrm{pH}$ of 5.3 and silica saturation index less than 0.5 would be assigned a $\mathrm{pH}$ grade of $\mathrm{C}$ and a silica content grade of $\mathrm{A}$.

Although the grades for each of the sub-attributes are independent, the rolled-up Fluid Chemistry character grade reported should be the sub-attribute that corresponds to the most severe outcomes.

Example: If the $\mathrm{pH}$ grade and silica content grade are $\mathrm{A}$ and the total dissolved solids content is $\mathrm{D}$ (the corrosivity is inhibiting the use of traditional tools), then report the rolled-up Fluid Chemistry character grade as $\mathrm{D}$.

When reporting Fluid Chemistry character grade, consider the conceptual model, activities performed, and quality of the data collected. Often, several activities and data sources can be used to understand fluid chemistry; greater confidence in estimates is obtained when independent observations yield similar results. Refer to the activity index and execution index sub-sections of this Fluid Chemistry Grade section for more details on activities used to evaluate fluid-chemistry characteristics.

\section{Activity Index}

The chemistry of reservoir brines and gases is interpreted through various analytical methods. The Activity Indices (Tables 14-1B through 14-6B) list activities ordered by their ability to represent fluid chemistry. The multiple sub-indices below reflect that an understanding of a system's chemistry is 
characterized by multiple factors, including $\mathrm{pH}$, bicarbonate, silica, and total dissolved solid concentrations.

Decisions on the most appropriate activity index to report should be done within the context of the conceptual model. This integrated approach, which incorporates all types of fluid chemistry data, is likely to better capture the heterogeneity in the reservoir. Although the indices for $\mathrm{pH}$, non-condensable gases, bicarbonate, total dissolved solids, and silica are independent, report for the Fluid Chemistry activity index the sub-attribute activity index that best represents the largest influence over the chemistry of the system.

Example: If the activity index for $\mathrm{pH}$ and Silica is $\mathrm{A}$ and Total Dissolved Solids is $\mathrm{B}$, with the limiting factor being the amount of scale, then the activity index should be reported as $B$.

If there is scientific reason to report other than as prescribed, it is important to note this discrepancy and the justification when reporting.

\section{Execution Index}

For each activity used to assess fluid chemistry, an Execution Index reflects how the data were verified relative to best practices. The index qualitatively indicates relative error and uncertainty in fluid chemistry-related exploration activities.

The tables below list the primary types of activities used to assess fluid geochemistry. There are two important factors to keep in mind:

- These tables are not an exhaustive list of all possible analytical methods that could be used to assess fluid chemistry. Those who have used (or wish to use) an analytical method or technique not listed here should contact the DOE GTO for guidance to create an appropriate execution index for reporting.

- The criteria in these tables are not meant to be guidelines to perform these exploration activities and are not an exhaustive list of all quality controls that must be performed. It is assumed that all analyses are performed by an experienced professional. These tables are for reporting purposes only.

For the selected "most representative" activity used to report fluid chemistry grade, select the execution index that best corresponds to the quality of information obtained.

Example: If chemistry was determined from titration, then use Table $14.2 \mathrm{~B}$ to determine the appropriate execution index (i.e., indicate how well the titrations were done). If information obtained by a contractor performing the titrations can be shown to meet all the criteria in $A$, report $A$ as the execution index value. 


\section{Sub-Attribute Character Grades and Activity Indices Tables}

The following tables provide descriptions of each sub-attribute grade. For each sub-attribute, select the most appropriate grade to describe the resource, and the associated activity and execution indices that describe how you arrived at the reported grade.

\section{Sub-Attribute 1: $\mathrm{pH}$}

$\mathrm{pH}$ drives the solubility of mineral species within geothermal fluid and can control the amount of species deposited when fluid is brought to the surface. The two most common scaling species (silica and calcium carbonate) are considered in sub-attributes 6 and 4, respectively.

Additionally, low-pH geothermal fluids can cause corrosion of steel, requiring careful material selection for casing, pipes, etc. (Brown 2013). Corrosion in plant infrastructure also depends on the content of corrosive gas content in geothermal fluids, which is addressed in sub-attribute 2 below.

Table 14-1A. Fluid Chemistry Sub-Attribute Grade: pH

\begin{tabular}{cll} 
Index & pH (at STP) & Description \\
\hline A & 7.0 & Ideal \\
\hline B & 5.6 to $<7$ or $>7$ to 8.4 & Favorable \\
\hline C & 4.5 to $<5.6$ or $>8.4$ to 10 & Challenging \\
\hline D & 3 to $<4.5$ or $>10$ to 11 & Difficult \\
\hline E & $<<3$ or $>11$ & Acidic/Caustic \\
\hline
\end{tabular}

Table 14-1B. Fluid Chemistry Sub-Attribute Activity: pH

\begin{tabular}{cll} 
Index & Activity (for $\mathrm{pH})$ & $\begin{array}{l}\text { See Related Execution } \\
\text { Index }\end{array}$ \\
A & $\begin{array}{l}\text { Probe - Reservoir fluid directly } \\
\text { sampled in field }\end{array}$ & Table 15-1 \\
\hline B & $\begin{array}{l}\text { Probe - Mixed reservoir fluid } \\
\text { sampled in field }\end{array}$ & Table 15-1 \\
\hline C & Paper test strips in field & Table 15-2 \\
\hline D & $\begin{array}{l}\text { Probe - Bottled reservoir fluid in } \\
\text { lab }\end{array}$ & Table 15-1 \\
\hline E & Probe - Bottled mixed fluid in lab & Table 15-1 \\
\hline
\end{tabular}


Sub-Attribute 2: Corrosive Gas Content $\left(\mathrm{HCl}, \mathrm{SO}_{2}\right.$, and/or $\left.\mathrm{H}_{2} \mathrm{~S}\right)$

When geothermal fluid chemistry causes corrosion of metals in production infrastructure, heat capture and energy production become more difficult and expensive (Nogara and Zarrouk 2018). Corrosion can be caused by a number of processes arising from the unique physio-chemical characteristics of a geothermal fluid. Facility designers must match equipment materials and design to geothermal fluid corrosive gas type and corrosion mechanism. This sub-attribute evaluates $\mathrm{HCl}, \mathrm{SO}_{2}$, and $\mathrm{H}_{2} \mathrm{~S}$ gas species by weight percent to account for variations in geothermal water chemistry between areas.

Table 14-2A. Fluid Chemistry Sub-Attribute Grade: Corrosive Gas Content $\left(\mathrm{HCl}, \mathrm{SO}_{2}\right.$, and/or $\left.\mathrm{H}_{2} \mathrm{~S}\right)$

\begin{tabular}{cll} 
Index & $\begin{array}{l}\text { Corrosive Gases } \\
\left(\mathrm{HCl}, \mathrm{SO}_{2} \text {, and/or } \mathrm{H}_{2} \mathrm{~S}\right)\end{array}$ & Description \\
\hline A & $0.0-0.001 \mathrm{wt} \%$ & Ideal \\
\hline B & $0.001-0.01 \mathrm{wt} \%$ & Favorable \\
\hline C & $0.01-0.025 \mathrm{wt} \%$ & Challenging \\
\hline D & $0.025-0.5 \mathrm{wt} \%$ & Difficult \\
\hline E & $>0.5 \mathrm{wt} \%$ & Corrosive \\
\hline
\end{tabular}

Table 14-2B. Fluid Chemistry Sub-Attribute Activity: Corrosive Gas Content

\begin{tabular}{|c|c|c|}
\hline Index & Activity (for Corrosive Gases) & See Related Execution Index \\
\hline A & $\begin{array}{l}\text { Titration - Reservoir gas } \\
\text { directly sampled in field }\end{array}$ & Table 15-3 \\
\hline B & $\begin{array}{l}\text { Titration - Mixed reservoir } \\
\text { gas sampled in field }\end{array}$ & Table 15-3 \\
\hline C & $\begin{array}{l}\text { Titration - Bottled reservoir } \\
\text { gas in lab }\end{array}$ & Table 15-3 \\
\hline D & $\begin{array}{l}\text { Titration - Bottled mixed gas } \\
\text { in lab }\end{array}$ & Table 15-3 \\
\hline E & Titration - Soil gas samples & Table 15-3 \\
\hline
\end{tabular}




\section{Sub-Attribute 3: Non-Condensable Gas Content}

Geothermal fluids typically contain several types of non-condensable gases (NCGs), which can significantly impact the performance of power generation and heating facilities (Khagani et al. 2013). Removing NCGs requires processes (e.g., vacuum pump, steam ejectors, degasification) that place parasitic loads on equipment and reduce plant efficiency. Depending on the NCG composition, gases such as $\mathrm{H}_{2} \mathrm{~S}$ may require removal consistent with applicable environmental and safety requirements.

Table 14-3A. Fluid Chemistry Sub-Attribute Grade: Non-Condensable Gas Content

\begin{tabular}{cll} 
Index & Non-Condensable Gases & Description \\
\hline A & $0-0.5 w t \%$ & Ideal \\
\hline B & $0.5-1 w t \%$ & Favorable \\
\hline C & $1-2.5 w t \%$ & Challenging \\
\hline D & $2.5-5.0 w t \%$ & Difficult \\
\hline E & $>5 w t \%$ & Inefficient \\
\hline
\end{tabular}

Table 14-3B. Fluid Chemistry Sub-Attribute Activity: Non-Condensable Gas Content

\begin{tabular}{cll} 
Index & Activity (for non-condensable gases) & $\begin{array}{l}\text { See Related } \\
\text { Execution } \\
\text { Index }\end{array}$ \\
A & Titration - Reservoir gas directly sampled in field & Table 15-3 \\
\hline B & Titration - Mixed reservoir gas sampled in field & Table 15-3 \\
\hline C & Titration - Bottled reservoir gas in lab & Table 15-3 \\
\hline D & Titration - Bottled mixed gas in lab & Table 15-3 \\
\hline E & Titration - Soil gas samples & Table 15-3 \\
\hline
\end{tabular}




\section{Sub-Attribute 4: Bicarbonate Content}

Extracting heat from geothermal fluid lowers the temperature of the fluid, which changes the solubility of dissolved species present in the fluid (Brown 2013). These species can form deposits within pipes and infrastructure. Scaling can create significant production issues in water-dominated hydrothermal fields by blocking pipes and reducing flow and/or heat-transfer efficiency in plant infrastructure (Utami et al. 2014; Brown 2013).

Calcite $\left(\mathrm{CaCO}_{3}\right)$ scaling can occur in geothermal production wells and production infrastructure, limiting productivity and requiring expensive remediation (Brown 2013). Dissolved carbon dioxide $\left(\mathrm{CO}_{2}\right) \mathrm{can}$ dissolve calcite into soluble calcium and bicarbonate ions, as shown in the equation below (Brown 2013):

$$
\mathrm{CaCO}_{3}+\mathrm{H}_{2} \mathrm{O}+\mathrm{CO}_{2} \Leftrightarrow \mathrm{Ca}^{++}+2 \mathrm{HCo}_{3}^{-}
$$

When fluid is extracted from the reservoir and the solubility of carbonate is decreased, this reaction is reversed, which results in the deposition of carbonate minerals such as calcite. The bicarbonate content grade is reported as the carbonate saturation index, which is derived from the solubility of carbonate minerals at the reported resource temperature, salinity, and pH. For details on calculating carbonate SI, see Fournier 1985.

Table 14-4A. Fluid Chemistry Sub-Attribute Grade: Bicarbonate Content

\begin{tabular}{cll} 
Index & Carbonate $\left(\mathrm{CaCO}_{3}\right) \mathrm{SI}^{3}$ & Description \\
\hline A & $<0$ & Undersaturated \\
\hline B & 0 or Unknown & Equilibrium \\
\hline C & $>0-1$ & Saturated \\
\hline D & $1-2$ & Slightly oversaturated \\
\hline E & $>2$ & $\begin{array}{l}\text { Highly oversaturated/ } \\
\text { scaling }\end{array}$ \\
\hline
\end{tabular}

Table 14-4B. Fluid Chemistry Sub-Attribute Activity: Bicarbonate Content

\begin{tabular}{cll} 
Index & Activity (for bicarbonate) & $\begin{array}{l}\text { See Related } \\
\text { Execution Index }\end{array}$ \\
\hline A & Titration - Reservoir fluid & Table 15-4 \\
\hline B & Calculation from analysis of Total $\mathrm{CO}_{2}$ & $\begin{array}{l}\text { Apply NCG sub- } \\
\text { attribute execution }\end{array}$ \\
\hline C & $\begin{array}{l}\text { Alkalinity - Immediate titration of } \\
\text { reservoir fluid in field }\end{array}$ & Table 15-4 \\
\hline D & Alkalinity - Titration of bottled fluid in lab & Table 15-4 \\
\hline
\end{tabular}

\footnotetext{
${ }^{3}$ where $\mathrm{SI}=\log (\mathrm{Q} / \mathrm{K})$

$\mathrm{Q}=\mathrm{aCa}^{2+} \mathrm{aCO}_{3}^{2-}$

$\mathrm{K}=$ equilibrium solubility at $\mathrm{T}, \mathrm{pH}$ conditions
} 


\section{Index Activity (for bicarbonate)}

E Calculated/assumed from $\mathrm{pH}$ alone
See Related

Execution Index

Apply pH subattribute execution

\section{Sub-Attribute 5: Total Dissolved Solid Content}

Geothermal fluids can contain different dissolved species and ions. Variations in water chemistry can affect different aspects of operations including the efficiency of heat capture from geothermal fluid, plant design, and disposal regulations for geothermal waters. This sub-attribute evaluates the total dissolved solid species in geothermal fluids in parts-per-million with the understanding that increased concentrations require more significant mitigation measures.

Table 14-5A. Fluid Chemistry Sub-Attribute Grade: Total Dissolved Solid Content

\begin{tabular}{|c|c|c|}
\hline Index & Total Dissolved Solids & Description \\
\hline A & $<1,000 \mathrm{ppm}$ & Ideal \\
\hline B & $1,000-<5,000 \mathrm{ppm}$ & Favorable \\
\hline C & $5,000-<20,000$ ppm & Challenging \\
\hline D & $20,000-100,000$ ppm & Difficult \\
\hline $\mathbf{E}$ & $>100,000$ ppm & Corrosive \\
\hline Index & Activity (for total dissolved solids) & $\begin{array}{l}\text { See Related Execution } \\
\text { Index }\end{array}$ \\
\hline A & $\begin{array}{l}\text { Conductivity Probe-Reservoir fluid } \\
\text { directly sampled in field }\end{array}$ & Table 15-5 \\
\hline B & $\begin{array}{l}\text { Field Measurements - Chloride } \\
\text { titrations }\end{array}$ & Table 15-6 \\
\hline C & $\begin{array}{l}\text { Conductivity Probe - Bottled } \\
\text { reservoir fluid in lab }\end{array}$ & Table 15-5 \\
\hline D & $\begin{array}{l}\text { Conductivity Probe - Mixed } \\
\text { reservoir fluid sampled in field }\end{array}$ & Table 15-5 \\
\hline $\mathbf{E}$ & $\begin{array}{l}\text { Fluid-inclusion freezing point } \\
\text { measurements }\end{array}$ & Table 15-7 \\
\hline
\end{tabular}




\section{Sub-Attribute 6: Silica Content}

Extracting heat from geothermal fluid lowers the temperature of the fluid, which reduces the solubility of dissolved species present within the fluid (Brown 2013). These species can form deposits within pipes and infrastructure. Scaling can create significant production issues in water dominated hydrothermal fields by blocking pipes and reducing flow and/or heat-transfer efficiency in plant infrastructure (Utami et al. 2014; Brown 2013).

Silica scaling in geothermal systems can result from the increase in amorphous silica solubility in geothermal fluids with high degrees of saturation. At high temperatures, quartz reaches equilibrium between solid and dissolved species via the following reaction (Brown 2013):

$$
\mathrm{SiO}_{2}(s)+2 \mathrm{H}_{2} \mathrm{O} \Leftrightarrow \mathrm{H}_{4} \mathrm{SiO}_{4}(a q)
$$

Silica scaling affects operations at many geothermal sites (Demir et al. 2014; Zarrouk et al. 2014). Silica scaling occurs if the geothermal fluid becomes oversaturated with respect to silica as it is cooling. Hence, the silica content grade is reported as the silica saturation index (SSI). SSI is defined as the ratio of the silica concentration in the brine divided by the equilibrium silica concentration for the silica mineral (e.g., amorphous silica, christobalite, chalcedony, quartz) stable at the reported resource temperature, salinity, and pH (Brown 2011). For details on calculating SSI, see Fournier 1985.

Table 14-6A. Fluid Chemistry Sub-Attribute Grade: Silica Content

\begin{tabular}{cll}
\hline Index & Silica Saturation Index $^{4}$ & Description \\
A & $<1$ & Undersaturated \\
\hline B & 1 or unknown & Equilibrium \\
\hline C & $>1-1.5$ & Saturated \\
\hline D & $1.5-2$ & Slightly oversaturated \\
\hline E & $>2$ & $\begin{array}{l}\text { Highly oversaturated/ } \\
\text { scaling }\end{array}$ \\
\hline
\end{tabular}

Table 14-6B. Fluid Chemistry Sub-Attribute Activity: Silica Content

\begin{tabular}{|c|c|c|}
\hline Index & Activity (for silica concentrations) & $\begin{array}{l}\text { See Related Execution } \\
\text { Index }\end{array}$ \\
\hline A & $\begin{array}{l}\text { Colorimeter - Molybdosilicate } \\
\text { method }\end{array}$ & Table 15-9 \\
\hline B & $\begin{array}{l}\text { Inductively coupled plasma-atomic } \\
\text { emission spectroscopy (ICP-AES) }\end{array}$ & Table $15-10$ \\
\hline C & $\begin{array}{l}\text { Inductively coupled plasma-mass } \\
\text { spectrometry (ICP-MS) }\end{array}$ & Table 15-11 \\
\hline D & $\begin{array}{l}\text { Atomic absorption spectrometry } \\
\text { (AAS) }\end{array}$ & Table $15-12$ \\
\hline $\mathbf{E}$ & Pocket colorimeter/test kit & Table 15-13 \\
\hline
\end{tabular}

\footnotetext{
${ }^{4} \mathrm{SSI}$ is calculated as the ratio of the silica concentration in the brine divided by the equilibrium amorphous silica solubility at the brine's $\mathrm{pH}$, temperature, and pressure conditions (Brown 2011).
} 


\section{Sub-Attribute Execution Indices Tables}

Table 15-1. Execution Index: Laboratory Analysis - pH

Corresponds to sub-attribute $\mathrm{pH}$, activity indices: $A, B, D, E$ (Tables 14-1A,B)

\section{Index Execution Details for Laboratory Analysis - pH}

A - Strict sampling protocols for rinsing and sealing bottles.

- All instruments calibrated daily.

- Calibration standards span the variation of the measured samples.

- Known temperature at which sample is taken, and temperature at time of lab analysis.

- Known whether sample has been degassed in the process.

B - Sampling protocols for rinsing and sealing bottles, implemented with some variation between researchers.

- All instruments calibrated at least once per week.

- All instruments operating within the analytical limits for majority of samples.

- Known temperature at which sample is taken, and temperature at time of lab analysis.

- Not known whether sample has been degassed in the process.

C - Results taken from previous third-party studies of the area (either literature or contractors) with little or limited information on survey methods, replication, or error.

D - No set sampling protocols for rinsing and sealing bottles specific to methods.

- No known or regularly scheduled calibration schedule.

- Analytical measurement limits are not appropriate for the majority of sample chemistries.

- Calibration standards do not span the variation of the measured samples.

- Neither temperature at which sample is taken nor temperature at time of lab analysis is recorded (or available).

- Not known whether sample has been degassed in the process.

E Assumed from studies of analogous geothermal settings or extrapolated from studies of nearby areas. 
Table 15-2. Execution Index: Chemistry Activities

Corresponds to sub-attribute $\mathrm{pH}$, activity index: $C$ (Tables 14-1A,B)

Index Execution Details for Activity: Paper pH test strips

A - Test strips stored in sealed, covered packages in a controlled climate out of sunlight.

- Excess water thoroughly removed before color comparison.

- Matching of test strip to color key occurred consistently after wait time prescribed by manufacturer.

- Strips have not expired.

B - Test strips stored in covered packages, but in sunlight or extreme temperature.

- Excess water only partially removed before color comparison.

- Matching of test strip to color key did not occur consistently after wait time prescribed by manufacturer.

C - Results taken from previous third-party studies of the area (either literature or contractors) with little or limited information on survey methods, replication, or error.

D - Test strips exposed to oxygen, sunlight, or extreme temperatures during storage.

- Excess water not removed before color comparison.

- Test strip matched immediately (i.e., no reaction time).

- Test strips have expired.

E - Assumed from studies of analogous geothermal settings or extrapolated from studies of nearby areas. 
Table 15-3. Execution Index: Titration

Corresponds to sub-attribute Corrosive Gases, Non-Condensable Gases activity indices: A, B, C, D, E (Tables 14-2A,B \& 14-3A,B)

Index Execution Details for Gas Content Titration

A - Gas and water phases measured; different phases were sampled with a miniseparator.

- Samples immediately acidified and titrated at the time of sampling/in the field.

- $\mathrm{pH}$ probe calibrated immediately prior to titration.

- Supplemented and/or corrected using reservoir field $\mathrm{pH}$ measurements.

- Validated by charge balance (separate sample measurements of cation and anion concentrations).

- Verified by gas chromatography.

B - Miniseparator has some leakage in capturing gas-liquid phase fractions; gas/steam ratios are not well constrained.

- Samples titrated within 24 hours.

- $\mathrm{pH}$ probe calibrated immediately prior to titration.

- Validated by charge balance (separate sample measurements of cation and anion concentrations) for selected locations.

C - Results taken from previous third-party studies of the area (either literature or contractors) with little or limited information on survey methods, replication, or error.

D - Only brine sample or fumarole gas sample collected, or gas/steam ratio unknown.

- Samples titrated after 24 hours.

- $\mathrm{pH}$ probe not calibrated immediately prior to titration.

- No additional supporting data.

E - Assumed from studies of analogous geothermal settings or extrapolated from studies of nearby areas. 
Table 15-4. Execution Index: Alkalinity Titration

Corresponds to sub-attribute Bicarbonate, activity indices: $A, C, D$ (Table 14-4A,B)

\section{Index Execution Details for Alkalinity Titration}

A - Samples immediately acidified.

- pH probe calibrated immediately prior to titration.

- Supplemented and/or corrected using reservoir field pH measurements.

- Validated by charge balance (separate sample measurements of cation and anion concentrations).

B $\quad$ Samples titrated within 24 hours.

- $\mathrm{pH}$ probe calibrated immediately prior to titration.

- Validated by charge balance (separate sample measurements of cation and anion concentrations) for selected locations.

C - Results taken from previous third-party studies of the area (either literature or contractors) with little or limited information on survey methods, replication, or error.

D - Samples titrated at some point after 24 hours.

- $\mathrm{pH}$ probe not calibrated immediately prior to titration.

- Samples may have cooled and degassed between collection and analysis

- No additional supporting data.

E - Assumed from studies of analogous geothermal settings or extrapolated from studies of nearby areas. 
Table 15-5. Execution Index: Conductivity Probe - Total Dissolved Solids (TDS)

Corresponds to sub-attribute Total Dissolved Solids, activity indices: $A, C, D$ (Table 14-5A,B)

\section{Index Execution Details for Conductivity Probe Measurements - TDS}

A - Gas and water phases measured; different phases were sampled with a miniseparator.

- Temperature, conductivity, and pH probes used within recommended operating temperatures.

- All probes calibrated in lab daily prior to fieldwork.

- All probes operating well within detection limits.

B - Miniseparator has some leakage in capturing gas-liquid phase fractions; gas/steam ratios are not well constrained.

- All probes calibrated at least once per week during fieldwork.

- All probes operating within detection limits for majority of sample locations.

C - Results taken from previous third-party studies of the area (either literature or contractors) with little or limited information on survey methods, replication, or error.

D - Only brine sample collected, or gas/steam ratio unknown.

- Temperature, conductivity, and pH probes used at top end of recommended operating temperatures.

- No known or regularly scheduled calibration schedule.

- Probe detection limits are not sensitive to majority of sample chemistries.

E - Assumed from studies of analogous geothermal settings or extrapolated from studies of nearby areas. 
Table 15-6. Execution Index: Chloride Titration

Corresponds to sub-attribute Total Dissolved Solids, activity index: $B$ (Table 14-5A,B)

\section{Index Execution Details for Chloride Titration - TDS}

A - Reagent recently purchased (e.g., within last 3 months).

- Aliquots added in small increments toward end of titration.

- Titration done immediately after sample taken.

- Measurements taken after downhole temperature probe readings stabilized.

- Results verified via test salt solution.

- Used within specified range (i.e., within detection limit)

B - Reagent near ( $\leq 3$ months) to end of shelf life.

- Aliquots added in small increments toward end of titration.

- Titration done within 2 and 12 hours after sample taken.

- Measurement downhole temperature probe readings not fully stabilized.

- Results not verified via test salt solution.

- Used within specified range (i.e., within detection limit)

C - Results taken from previous third-party studies of the area (either literature or contractors) with little or limited information on survey methods, replication, or error.

D $\quad$ Reagent older than shelf life/expired.

- Aliquots added in equal increments (not modified toward end of titration).

- Titration done $>12$ hours after sample taken.

- Measurement downhole temperature probe readings not stabilized.

- Results not verified via test salt solution.

- Results extrapolated outside of titrator's detection limit

E Assumed from studies of analogous geothermal settings or extrapolated from studies of nearby areas. 
Table 15-7. Execution Index: Fluid Inclusions - TDS

Corresponds to sub-attribute Total Dissolved Solids, activity index: E (Table 14-5A,B)

\section{Index Execution Details for Fluid Inclusions - TDS}

A - Heating-freezing table is appropriately and regularly calibrated for geothermal fluid ranges (daily or weekly, depending on frequency of use).

- Temperature increases/decreases are performed gradually (i.e., $0.1^{\circ}-0.2^{\circ} \mathrm{C} / \mathrm{min}$ ) for high resolution.

- Multiple large inclusions are used, allowing thorough testing.

- Fluid-inclusion results are interpreted in relation to the present-day hydrothermal system.

B - Heating-freezing table is appropriately calibrated for geothermal fluid ranges, but not recently (e.g., not within the most recent 6 months).

- Temperature increases/decreases are performed gradually (i.e., $0.1^{\circ}-0.2^{\circ} \mathrm{C} / \mathrm{min}$ ) only when nearing target temperature ranges and are otherwise $0.5^{\circ} \mathrm{C} / \mathrm{min}$.

- Most inclusions ( $>50 \%$ ) are too small for measurement, but some ( $>25 \%$ ) can be analyzed.

- Fluid-inclusion results are interpreted in relation to the present-day hydrothermal system.

C - Results taken from previous third-party studies of the area (either literature or contractors) with little or limited information on survey methods, replication, or error.

D - Heating-freezing table is not calibrated for geothermal fluid ranges.

- Temperature increases/decreases are not performed gradually (i.e., $>0.5^{\circ} \mathrm{C} / \mathrm{min}$ ) and do not target the desired temperature range.

- Most inclusions (>75\%) are too small for measurement or have leaked their gas phase.

- Fluid-inclusion results are interpreted without careful consideration of different hydrothermal episodes, i.e., fluid inclusion data may not be related to present-day hydrothermal system.

E - Assumed from studies of analogous geothermal settings or extrapolated from studies of nearby areas. 
Table 15-8. Execution Index: Colorimeter - Molybdosilicate Method

Corresponds to sub-attribute Silica, activity index: $B$ (Table 14-6A,B)

Index Execution Details for Colorimeter - Molybdosilicate Method

A $\quad$ Reagent stored in polyurethane bottles.

- Dilution within calibration range for all runs.

- Calibration done on frequent, regular schedule.

- Standards measured at or near face value.

B - Reagent stored in polyurethane bottles.

- Dilution within calibration range for most runs.

- Calibration done on regular, but not frequent, schedule.

- Standards measured at or near face value.

C - Results taken from previous third-party studies of the area (either literature or contractors) with little or limited information on survey methods, replication, or error.

D $\quad$ Reagent stored in glass bottles.

- Calibration done on regular, but not frequent, schedule.

- Not diluted to within calibration range.

- Standards significantly deviate from face value.

E Assumed from studies of analogous geothermal settings or extrapolated from studies of nearby areas.

Table 15-9. Execution Index: Inductively Coupled Plasma Atomic Emission Spectroscopy (ICP-AES)

Corresponds to sub-attribute Silica, activity index: B (Table 14-6A,B)

Index Execution Details for ICP-AES

A Dilution within calibration range for all runs.

- Calibration done on frequent, regular schedule.

- Standards measured at or near face value.

B - Dilution within calibration range for most runs.

- Calibration done on regular, but not frequent, schedule.

- Standards measured at or near face value.

C - Results taken from previous third-party studies of the area (either literature or contractors) with little or limited information on survey methods, replication, or error.

D $\quad$ Calibration done on regular, but not frequent, schedule.

- Not diluted to within calibration range.

- Standards significantly deviate from face value.

E Assumed from studies of analogous geothermal settings or extrapolated from studies of nearby areas. 
Table 15-10. Execution Index: Inductively Coupled Plasma Mass Spectrometry (ICP-MS)

Corresponds to sub-attribute Silica, activity index: C (Table 14-6A,B)

Index Execution Details for ICP-MS

A Dilution within calibration range for all runs.

- Calibration done on frequent, regular schedule.

- Standards measured at or near face value.

B - Dilution within calibration range for most runs.

- Calibration done on regular, but not frequent, schedule.

- Standards measured at or near face value.

C - Results taken from previous third-party studies of the area (either literature or contractors) with little or limited information on survey methods, replication, or error.

D Calibration done on regular, but not frequent, schedule.

- Not diluted to within calibration range.

- Standards significantly deviate from face value.

E Assumed from studies of analogous geothermal settings or extrapolated from studies of nearby areas. 
Table 15-11. Execution Index: Atomic Absorption Spectrometry (AAS)

Corresponds to sub-attribute Silica, activity index: $D$ (Table 14-6A,B)

\section{Index Execution Details for AAS}

A Dilution within calibration range for all runs.

- Calibration done on frequent, regular schedule.

- Standards measured at or near face value.

B - Dilution within calibration range for most runs.

- Calibration done on regular, but not frequent, schedule.

- Standards measured at or near face value.

C - Results taken from previous third-party studies of the area (either literature or contractors) with little or limited information on survey methods, replication, or error.

D $\quad$ Calibration done on regular, but not frequent, schedule.

- Not diluted to within calibration range.

- Standards significantly deviate from face value.

E - Assumed from studies of analogous geothermal settings or extrapolated from studies of nearby areas. 
Table 15-12. Execution Index: Pocket Colorimeter

Corresponds to sub-attribute Silica, activity index: $D$ (Table 14-6A,B)

Index Execution Details for Pocket Colorimeter

A - Reagent recently purchased (e.g., within 2 weeks).

- Matching of sample to color key occurred consistently after wait time prescribed by manufacturer.

- Sample acidified or diluted immediately.

B - Reagent older than half of its 2-month shelf life.

- Matching of sample to color key did not occur consistently after wait time prescribed by manufacturer.

- Sample acidified or diluted immediately.

C - Results taken from previous third-party studies of the area (either literature or contractors) with little or limited information on survey methods, replication, or error.

D $\quad$ Reagent older than the 2-month shelf life.

- Matching of sample to color key occurred immediately.

- Sample not acidified or diluted.

E Assumed from studies of analogous geothermal settings or extrapolated from studies of nearby areas. 


\section{GLOSSARY OF TERMS}

Activity:

Activity Index: Qualitative ranking of activities used to assign the character grade appropriate for each attribute.

Activity Threshold: Minimum exploration activities required to qualify for the next-most-advanced project readiness-level category.

Attribute:

Character:

Character Grade: Intrinsic measurement that best describes a key characteristic of the geothermal reservoir.

Execution:

Execution Index: Compares the diligence with which the technique was executed for the activity.

Fluid Availability: The accessibility and sustainability of fluid that can be used to transport heat from the reservoir.

Geological Grade: A set of attributes describing the quality of the geothermal resource that are associated with geological constraints.

Permeability: $\quad$ The ability, or measurement of a rock's ability, to transmit fluids and access heat.

\section{Project Readiness}

Level:

A set of terminology that differentiates stages of exploration activities by their geologic, technologic, and socioeconomic features and that differentiates these stages by activity threshold.

Resource Grade: The quality of the geothermal resource as it relates to the potential to extract heat, determined as a combination of resource attributes.

Resource Size: The quantity of recoverable heat for a geothermal resource. 


\section{Socioeconomic}

Grade:

A set of attributes describing the quality of the geothermal resource that external to the resource itself and the technologies used to extract heat.

Technical Grade: A set of attributes describing the quality of the geothermal resource that are associated with producing a useable heat resource.

Temperature: In situ temperature, enthalpy, and fluid phase of the reservoir, which indicate the quality of heat available for extraction.

Volume: $\quad$ Volumetric size of the heat reservoir (via assessments of thickness and area), which indicates the quantity of available heat to be extracted. 


\section{REFERENCES}

Anderson, E., Crosby, D. and Ussher, G. 2000. Bull's Eye! - Simple resistivity imaging to reliably locate the geothermal reservoir. Proceedings World Geothermal Congress 2000, p. 909-914.

Árnason, K., Vilhjálmsson, A. M., \& Björnsdóttir, T. 2009. A Study of the Krafla Volcano Using Gravity, Micro Earthquake and MT Data. Short Course IV on Exploration for Geothermal Resources. Lake Naivasha, Kenya: United Nations University - Geothermal Training Programme.

Atmoko, F. A. (n.d.). Predicition of Geothermal Manifestation Area Using ASTER Imagery in Northern Sukabumi, West Java, Indonesia. http://www.academia.edu/2053946/Prediction_of Geothermal Manifestation_Area in Northern Sukabumi West Java Indonesia.

Axelsson, G. and Gunnlaugsson, E. 2000. Background: Geothermal utilization, management and monitoring, in Long-term monitoring of high- and low-enthalpy fields under exploitation. World Geothermal Congress 2000 Short Course, Japan, p. 3-10

Barton, C. A., Zoback, M.D., and Moos, D., 1995, Fluid flow along potentially active faults in crystalline rock. Geology, v. 23, p. 683-686.

Bendritter, Y., and Cormy, G. 1990. Possible approach to geothermal research and relative costs, in Dickson, M.H., and Fanelli, M., eds., Small Geothermal Resources: A Guide to Development and Utilization. UNITAR, New York, p. 59-69.

Blewitt, G., and Coolbaugh, M. 2002. Targeting of Potential Geothermal Resources in the Great Basin from Regional Relationships between Geodetic Strain and Geologic Structures. Transactions Geothermal Resources Council. V. 26, p. 523-526.

Brown, K., 2013. Mineral scaling in geothermal power production (No. 39). United Nations University, Geothermal training programme.

Brown, K. 2011. Thermodynamics and kinetics of silica scaling. Proceedings International Workshop on Mineral Scaling. Manila, Philippines, 25-27, 2011.

Browne, P. 1978. Hydrothermal Alteration in Active Geothermal Fields. Annual Review of Earth and Planetary Sciences, v. 6, p. 229-248.

Cloetingh, S. and J. Negendank (eds.) 2010. New Frontiers in Integrated Solid Earth Sciences, International Year of Planet, p. 261

Cumming, W.B. 2009a. Geothermal resource conceptual models using surface exploration data: Proceedings, $34^{\text {th }}$ Workshop on Geothermal Reservoir Engineering, Stanford University, 6 p.

Cumming, W.B. 2009b. A conceptual model approach to the geophysical exploration of permeable geothermal reservoirs that considers context and uncertainty. SEG Houston 2009 International Exposition and Annual Meeting, extended abstract.

Cumming, W., and Mackie, R. 2010. Resistivity imaging of geothermal resources using 1D, 2D, and 3D MT inversion and TDEM static shift correction illustrated by a Glass Mountain case history. Proceedings World Geothermal Congress 2010, $10 \mathrm{p}$.

Curewitz, D., \& Karson, J. A. 1997, December. Structural settings of hydrothermal outflow: Fracture permeability maintained by fault propagation and interaction. Journal of Volcanology and Geothermal Research, v. 79(3-4), p. 149-168.

Demir, M.M., Baba, A., Atilla, V., Inanlı, M., 2014. Types of the scaling in hyper saline geothermal system in northwest Turkey. Geothermics, v. 50, p. 1-9. https://doi.org/10.1016/j.geothermics.2013.08.003

DiPippo, Ronald. 2004. Second Law assessment of binary plants generating power from low-temperature geothermal fluids. Geothermics, v. 33, p. 565-586.

Einarsson, P. 1978. S-wave shadows in the Krafla caldera in NE-Iceland, evidences for a magma chamber in the crust. Bulletin of Volcanology, v. 41(3), p. 1-9. 
Faulds, J., Hinz, N., Coolbaugh, M., Cashman, P., Kratt, C., Dering, G., Edwards, J., Mayhew, B., and McLachlan, H. 2011. Assessment of Favorable Structural Settings of Geothermal Systems in the Great Basin, Western USA. Geothermal Resources Council Transactions, v. 35, 777-783.

Fournier, R.O. 1985. "The behavior of silica in hydrothermal solution." Reviews in Economic Geology, v. 2 p. $45-72$.

Fournier, R.O. 1991. Water geothermometers applied to geothermal energy. In. "Application of geochemistry in geothermal reservoir development." UNITAR/UNDP center on small energy resources, Rome, Italy, 1991, pp. 37-69.

Fournier, R.O. 1992. Water geothermometers applied to geothermal energy, Applications of Geochemistry in Geothermal Reservoir Development, Series of Technical Guides on the Use of Geothermal Energy, Rome, Italy: UNITAR/UNDP Cent on Small Energy Resources, p. 119-144.

Georgsson, L. S. 2009. Geophysical Methods In Geothermal Exploration. Short Course IV on Exploration for Geothermal Resources (p. 16). Lake Naivasha, Kenya: United Nations University-Geothermal Training Programme.

Georgsson, L.S., and Fridleifsson, G.Ó. 2000. The geothermal exploration of the Öxarfjördur high temperature area, NE-Iceland. Proceedings of the World Geothermal Congress 2000, Kyushu-Tohoku, Japan, p.1157-1162.

Giggenbach, W.F. 1988. Geothermal solute equilibria. Derivation of Na-K-Mg-Ca geoindicators, Geochimica et Cosmochimica Acta, v. 52(12), p. 2749-2765.

Giggenbach, W.F. and Goguel, R.L. 1989. Collection and analysis of geothermal and volcanic water and gas discharges, DSIR report CD 2401, $4^{\text {th }}$ ed., Petone, New Zealand.

Grant, M.A. 2014. Stored-heat assessments: a review in the light of field experience. Geoth. Energ. Sci., v. 2, p. 4954.

Gupta, H. 1980. Geothermal Resources: An Energy Alternative. Elsevier.

Hanna, W.F., Hassemer, J.H., Elliott, J.E., Wallace, C.A., and Snyder, S.L. 1994. Maps Showing Gravity and Aeromagnetic Anomalies in the Butte $1 \times 2$ Quadrangle, Montana. U.S. Department of the Interior and U.S. Geological Survey. 2050i. http://pubs.usgs.gov/imap/2050i/report.pdf

Haselwimmer, C., Prakash, A., and Holdmann, G. 2013. Quantifying the heat flux and outflow rate of hot springs using airborne thermal imagery: Case study from Pilgrim Hot Springs, Alaska. Remote Sensing of Environment. v. 136, p. 37-46.

Henley, R.W., 1983. pH and silica scaling control in geothermal field development. Geothermics 12, $307-321$. https://doi.org/10.1016/0375-6505(83)90004-4

Henley, R. W., and Ellis, A. 1983. Geothermal Systems Ancient and Modem, A Geochemical Review, Earth Sciences Review, v. 19, p. 1-50.

Henley, R.W., Truesdell, A.H., and Barton, P.B., Jr. 1984. Fluid-mineral equilibria in hydrothermal systems. Reviews in Economic Geology, v. 1, Society of Economic Geologists, 267 p.

Hickman, S., Barton, C.A., Zoback, M.D., Morin, R., Sass, J., and Benoit, R., 1997, In situ stress and fracture permeability along the Stillwater fault zone, Dixie Valley, Nevada: International Jour. of Rock Mechanics and Mining Science, v. 34, p. 4-14.

Hochstein, M.P. 1988. Assessment and modeling of geothermal reservoirs (small utilization schemes). Geothermics, v. 17, n. 1, p. 15-49.

Horne, R. 1990. Modern Well Test Analysis: A Computer-Aided Approach. Stanford University.

Karlsdóttir, R., Vilhjálmsson, A., Árnason, K., \& Beyene, A. 2012. Peistareykir Geothermal Area, Northern Iceland 3D Inversion of MT and TEM Data (p. 173). Reykjavík.

Lyatsky, H. 2010, October. Magnetic and Gravity Methods in Mineral Exploration: the Value of Well-Rounded Geophysical Skills. CSEG Recorder, p. 31-35. http://csegrecorder.com/articles/view/magnetic-and-gravitymethods-in-mineral-exploration

Kaya, E., Zarrouk, S.J., O'Sullivan, M.J. 2011. Reinjection in geothermal fields: A review of worldwide experience. Renewable and Sustainable Energy Reviews, v. 15, p. 47-68. 
Khaghani, A., Date, A., Akbarzadeh, A., 2013. Sustainable removal of non-condensable gases from geothermal waters. Renewable and Sustainable Energy Reviews, v. 21, p. 204-214. https://doi.org/10.1016/j.rser.2012.12.001

Kharaka, Y.K, Gunter, W.D., Aggarwal, P.K., Perkins, E.H., and DeBraal, J.D. 1988. SOLMINEQ88: A Computer Program for Geochemical Modeling of Water-Rock Interactions, U.S. Geological Survey Water-Resources Investigations Report 88-4227.

Muffler, L.P.J., and Cataldi, R. 1978. Methods for regional assessment of geothermal resources. Geothermics. v. 7, p. 53-89.

Neupane, G., Baum, J.S., Mattson, E.D., Mines, G.L., Palmer, C.D., and Smith, R.W. 2015. Validation of multicomponent equilibrium geothermometry at four geothermal power plants. Proceedings, $40^{\text {th }}$ Workshop on Geothermal Reservoir Engineering, Stanford University, Stanford, CA, SGP-TR-204, 17 p.

Nicholson, K. N. 1993. Geothermal Fluids. Chemistry and Exploration Techniques. 263 p.

Nogara, J., Zarrouk, S.J., 2018. Corrosion in geothermal environment: Part 1: Fluids and their impact. Renewable and Sustainable Energy Reviews, v. 82, p. 1333-1346. https://doi.org/10.1016/j.rser.2017.06.098

Pálmason, G. 1967. On heat flow in Iceland in relation to the Mid-Atlantic Ridge. In: Björnsson S (ed.), Iceland and Mid-Ocean Ridges, Soc. Sci. Islandica

Peiffer, L., Wanner, C., Spycher, N., Sonnenthal, E.L., Kennedy, B.M., and lovenitti, J. 2014. Optimized multicomponent vs. classical geothermometry: Insights from modeling studies at the Dixie Valley geothermal area. Geothermics, v. 51, p. 154-169.

Reed, M.J, and Mariner, R.H. 1991. Quality Control of Chemical and Isotopic Analyses of Geothermal Water Samples, Proceedings, $16^{\text {th }}$ Workshop on Geothermal Reservoir Engineering, Stanford University, SGP-TR-134, p. 9-13.

Renner, J.L., White, D.E., and Williams, D.L. 1975. Hydrothermal convection systems. In: Assessment of Geothermal Resources of the United States - 1975, D.E White and D.L. Williams, eds. U.S. Geological Survey Circular 726, p. 5-57.

Sanyal, S.K. 2005. Classification of Geothermal Systems: A Possible Scheme. In: Thirtieth Workshop on Geothermal Reservoir Engineering; 2005/02/02; Stanford, California. Stanford, California: Stanford University; SGP-TR-176, p. 8.

Spycher, N., Peiffer, L., Sonnenthal, E.L., Saldi, G., Reed, M.H., and Kennedy, B.M. 2014. Integrated multicomponent solute geothermometry. Geothermics, v. 51, p. 113-123.

Spycher, N., Finsterele, S., and Dobson, P. 2016. New Developments in Multicomponent Geothermometry. $41^{\text {st }}$ Workshop on Geothermal Reservoir Engineering, Stanford University, Stanford, CA, SGP-TR-204, 17 p.

Utami, W.S., Herdiantita, N.R., Atmaja, R.W., 2014. The effect of temperature and pH on the formation of silica scaling of Dieng geothermal field, Central Java, Indonesia, in: Thirty-Ninth Workshop on Geothermal Reservoir Engineering. Stanford University.

Walters, M.A., and Combs, J. 1992. Heat flow in the Geysers-Clear Lake geothermal area of Northern California, U.S.A. Geothermal Resources Council Monograph on The Geysers Geothermal Field, Special Report No. 17, p. 43-53.

Williams, C.F., Reed, M.J., and Mariner, R.H. 2008. A review of methods applied by the U.S. Geological Survey in the assessment of identified geothermal resources. USGS Open-File Report 2008-1296, 27 p.

Williams, C. and DeAngelo, J. 2011. Evaluation of Approaches and Associated Uncertainties in the Estimation of Temperatures in the Upper Crust of the Western United States. GRC Transactions, v. 35, p. 1599-1605.

Williams, C.F. 2014. Evaluating the volume method in the assessment of identified geothermal references. Geothermal Resources Council Transactions, v. 38, p. 967-974.

Wohletz, K., \& Heiken, G. 1992. Appendix F: Core and Drill Cuttings: Geothermal Well Logs. In Volcanology and Geothermal Energy. Berkeley: University of California Press. http://publishing.cdlib.org/ucpressebooks/view?docld=ft6v19p151\&chunk.id=d0e26460\&toc.depth=1\&brand= $\underline{\text { ucpress }}$ 
Zarrouk, S.J., Woodhurst, B.C., Morris, C., 2014. Silica scaling in geothermal heat exchangers and its impact on pressure drop and performance: Wairakei binary plant, New Zealand. Geothermics, v. 51, p. 445-459. https://doi.org/10.1016/j.geothermics.2014.03.005 
APPENDIX A: SCIENTIFIC BACKGROUND TO RESOURCE GRADE

\section{TEMPERATURE}

\section{DOWNHOLE TEMPERATURE PROBE}

Measured downhole temperatures from probe readings are most likely of all techniques to provide accurate estimates of the in situ reservoir temperature, because the probe is directly measuring reservoir conditions. Reservoir temperatures can vary significantly within the geothermal reservoir from the main deep upflow zones to shallower outflow plumes, so there likely will be a range of measured reservoir temperatures from any given resource. Temperatures obtained from the productive zones are often the most representative of the reservoir.

The measured temperature profiles for a well can vary depending on whether the temperatures were recorded immediately after drilling (prior to thermal equilibration), under static conditions, or under flowing conditions. A downhole temperature log will be able to resolve differences in temperature for multiple fluid entries within a reservoir. Horner plot analyses can be used to correct for unequilibrated temperature measurements (Horne 1990).

\section{GEOTHERMOMETERS}

Before exploration wells have been drilled, the best way to estimate subsurface reservoir temperatures is by using chemical geothermometers. There are five general types of chemical geothermometers:

1. Silica geothermometers

2. Cation geothermometers

3. Gas geothermometers

4. Isotope geothermometers

5. Multicomponent geothermometers.

\section{Cation Geothermometers}

The quality of the fluid samples is critical to the predictive ability of the analysis. For example, the USGS geothermal resource assessment quantifies the reliability of water chemical analyses used for its calculation of cation geothermometers by calculating the ratio of the sample's charge balance to its error (Williams et al. 2008; Reed and Mariner 1991). Due to the assumptions involved in each, the choice of an appropriate geothermometer is also significant to the uncertainty of the temperature estimates. As Williams et al. (2008) summarize, silica geothermometers are appropriate only for fluids with pH between 5 and 7 (Fournier 1992); in more alkaline solutions, silica saturation should instead be evaluated via a computer model (Kharaka et al. 1988). The Giggenbach ternary K-Na-Mg cation diagram quickly allows an evaluation of whether a fluid has equilibrated, and the $\mathrm{K}-\mathrm{Mg}$ geothermometer provides additional information about fluid equilibration and/or mixing with shallow groundwater due to its consistent inverse relationship to temperature with depth, unless the fluids are chlorine-rich or magnesium data are unavailable or undetectable (Giggenbach 1988; Brook et al. 1979). In these cases, the $\mathrm{Na}-\mathrm{K}-\mathrm{Ca}$ geothermometer is recommended (Williams et al. 2008). As shown by the discrepancy in previous USGS geothermal resource assessments, the discontinuity between the lower temperature $\left(<100^{\circ} \mathrm{C}\right)$ and higher temperature $\left(>100^{\circ} \mathrm{C}\right)$ formulations of the widely used $\mathrm{Na}-\mathrm{K}-\mathrm{Ca}-\mathrm{Mg}$ cation 
geothermometers caused an underreporting of U.S. geothermal resources within $100^{\circ} \mathrm{C}$ and $130^{\circ} \mathrm{C}$ (Williams et al. 2008).

\section{Multicomponent Geothermometers}

Multicomponent geothermometers (such as GeoT and RTEst) have been developed to use the complete information contained in a geothermal fluid analysis (Spycher et al. 2014; Peiffer et al. 2014; Neupape et al. 2015). These geothermometers require the selection of an alteration mineralogy assemblage with which the fluid has equilibrated. When coupled with geochemical modeling tools (such as iTOUGH or PEST), they can be used to estimate the effects of mixing, boiling, and the concentrations of minor (yet important) fluid components, such as Al, Fe, and $\mathrm{Mg}$. Therefore, intelligent corrections to fluid composition are more readily apparent with multicomponent analyses (Spycher et al. 2016). These analyses require careful attention to all sources of uncertainty in each of the individual mineral-fluid reactions (and the selection of an appropriate thermodynamic database and subsurface mineral assemblage). The coherence in multicomponent methods may lead to a false sense of precision if the underlying assumptions are inappropriate (Spycher et al. 2014).

\section{Gas Geothermometers}

Gas geothermometers are derived from a range of empirical relationships observed in field studies, or based on temperature-dependent reactions governed by thermodynamics (such as the Fisher-Tropsch reaction). Given that in many cases (such as with gas samples obtained from surface features), the gassteam ratio is not known, and thus, the reactions are often cast as ratios of reactive gases with inert gases (such as $\mathrm{H}_{2} / \mathrm{Ar}$ vs. $\mathrm{CO}_{2} / \mathrm{Ar}$ )( Giggenbach and Goguel 1989). Where gas samples have reacted with shallow waters (such as in the case of gases bubbling from hot springs), some gas species may be preferentially stripped (such as $\mathrm{H}_{2} \mathrm{~S}$ and $\mathrm{NH}_{3}$ ), making their interpretation more challenging. In the case where there are no deep-sourced spring waters (such as areas with fumaroles and steam-heated bicarbonate and acid-sulfate springs), gas chemistry may provide the best method for estimating reservoir temperatures.

\section{THERMAL GRADIENT HOLE LOGS OF TEMPERATURE}

When geothermometry estimates are inconsistent between geothermometers, or in the case of a blind geothermal prospect where there are no surface thermal features and no deep wells, estimates of potential reservoir temperatures can be made using temperature-gradient wells. Thermal-gradient hole (TGH) temperatures are extrapolated using observed thermal gradients near the bottom of the hole to the estimated depth of the reservoir to estimate a resource temperature. These extrapolations bring potential for significant uncertainty and error, as discussed in the execution index.

TGH logs provide temperature conditions of the near surface. Bottom-hole temperatures are typically extrapolated at the same gradient to the estimated reservoir depth. Extrapolation of these results must be done with caution, and the uncertainties of estimates need to be considered. Thermal gradients are not always constant with depth and can change abruptly with changes in rock types (because different rocks have different thermal conductivities) and shifts from conductive to convective heat transfer. These changes can result in a shift to lower thermal gradients, near-isothermal conditions, or even a reversal to lower-temperature conditions at depth (in the case of a shallow outflow plume). A maximum temperature can be estimated using the boiling point-depth curve: for this method, a temperature of $100^{\circ} \mathrm{C}$ (or the temperature for boiling at the corresponding elevation) would be assigned to the top of the water column, and increasing temperatures with depth would be calculated using the increase in hydrostatic head pressure and the salinity of the fluid (Henley et al. 1984). 
MINERAL ASSEMBLAGE ESTIMATES OF TEMPERATURE

The combination of mineral formation temperature ranges, cross-cutting mineral relationships, and mineral assemblages is a powerful tool for constraining the temperature of the geothermal reservoir (Henley and Ellis 1983). Nevertheless, as since multiple episodes of hydrothermal alteration can be present in core or thin section, sometimes with overprinting retrograde alteration, using mineral assemblages as temperature constraints requires some knowledge of previous metamorphic and/or hydrothermal episodes. Formation temperatures can easily be inferred as higher than what actually exists if retrograde metamorphism is not properly identified because these techniques assume the most recent alteration is representative of the temperature of the active resource (Henley and Ellis 1983). Fluid-inclusion studies can provide constraints on trapping temperatures and fluid salinities.. These data need to be interpreted within the context of the paragenesis of the mineral assemblages in which they occur. Also, different lithologies can result in the generation of distinct alteration mineral assemblages at the same temperature.

\section{REGIONAL HEAT FLOW OF TEMPERATURE}

Regional heat-flow data are even less representative because they provide only an indication of heat reaching the surface, which is not representative of the reservoir conditions. Because regional heat flow is an extrapolation of downhole temperature measurements across a geospatial area, data processing and the resolution of the mapping are primary concerns (Williams and DeAngelo 2011).

\section{REMOTE SENSING AND SHALLOW TEMPERATURE PROBES}

Although not indicative of temperature at depth, estimates of heat flux from airborne thermal infrared remote sensing are more complete estimates of temperature variation than field observations alone because they account for thermal emanations between visible surface manifestations (Haselwimmer et al. 2013). Surface maps of two-meter probe measurements have also been used to indicate zones with shallow elevated heat flow, but do not provide direct insights into deep reservoir temperatures.

\section{SURFACE MANIFESTATIONS}

Surface manifestations of underlying geothermal activity include hot springs, fumaroles, and elevated ground temperatures. Because temperature anomalies may vary spatially and temporally in depth and horizontal extent, evaluating all of the temperature data across the entire geothermal system is crucial to assessing potential variability. For example, a typical hydrothermal system's surface manifestations may change from active to inactive over time and may be affected by external processes such as seasonal snow melt and irrigation. Because these features are discrete points in a larger geothermal area, mapping of geothermal manifestations only indicates the minimum likelihood of a resource, and it does not in itself identify resource temperature without sampling. The presence of surface thermal features also indicates the presence of enhanced permeability that permits these fluids to migrate upwards to the surface. 


\section{VOLUME}

\section{DEEP WELLS AND FLOW TESTING}

Well flow testing is the most accurate technique for estimating reservoir volume of because it provides the most direct information about the geothermal system. The most reliable method to determine the areal extent of a geothermal reservoir is by drilling the reservoir to determine the extent of the thermal anomaly and the flow of fluid. Once a resource has been discovered, step-out wells are typically drilled to confirm the areal extent of the field. Once several successful wells have been drilled, long-term flow tests can be conducted to evaluate interference between wells through pressure build-up and drawdown and to determine the sustainability of production from the field. The best information used to constrain estimates of reservoir thickness estimates are also derived from drilling, because they provide direct evidence of the vertical temperature profile, the extent of hydrothermal alteration, and location of fluid entries within the reservoir. When measured, the top of the reservoir is marked by the depth of the reservoir's clay cap seal, and the bottom of the reservoir is noted by the depth of sharp decreases in permeability.

Many geothermal fields are delineated by a central cluster of productive wells that encounter elevated temperatures and permeability, with marginal conditions encountered on the periphery of the field. Although highly reliable, it is often cost prohibitive to complete well tests at the number of locations needed to represent this variability. As an alternative, temperature-gradient data from distributed deep wells can also be used to constrain the areal limits of the geothermal anomaly (Walters and Combs 1992).

\section{THERMAL-GRADIENT HOLE LOGS}

The nature of the thermal gradient is another common way of identifying the location and thickness of the reservoir section. In a reservoir with sufficient permeability, advective transport of heat and fluid results in a near-isothermal temperature profile (also known as a convective thermal gradient). The thickness of this gradient can be used to estimate the thickness of the geothermal reservoir. ${ }^{5}$

\section{MAGNETOTELLURICS (MT)}

One of the most common methods employed to estimate the areal extent of a geothermal system during exploration is to conduct electrical geophysical surveys such as MT to delineate the depth and extent of a hydrothermal clay cap. MT methods measure the Earth's electromagnetic response to its naturally fluctuating magnetic field-a response governed by variations in rock electrical conductivity, which itself is a function of lithology, temperature, and fluid content. This allows one to assess either (or both) deep and upper crustal structures, depending on the frequency range chosen. A highly conductive clay cap is found in many geothermal systems and directly overlies and bounds the geothermal reservoir (Anderson et al. 2000; Cumming 2009a; Cumming 2009b; Cumming and Mackie 2010). The presence of a high-conductivity zone over the prospective geothermal reservoir could also represent the presence of fossil hydrothermal alteration instead of the presence of clay-rich sediments. Hence, this geophysical

\footnotetext{
${ }^{5}$ In some instances, viable hydrothermal systems can have conductive thermal gradients (such as seen in the high-temperature reservoir of the NW Geysers field), but these gradients are indicative of low-permeability conditions.
} 
signature is not definitive and needs to be interpreted in conjunction with other geologic, geophysical, and geochemical data.

\section{TRANSIENT ELECTROMAGNETICS (TEM)}

Like the MT method, the TEM method is based on electromagnetic interrogation of the subsurface, but instead uses engineered transmitter antennas for a source in lieu of Earth's natural magnetic field fluctuations. A large horizontal loop with a known current waveform is commonly used; however, grounded electric dipole antennas have also been investigated. One advantage of the loop antenna is the ability to deploy it from airborne (fixed-wing aircraft or helicopters) platforms, thus allowing for rapid reconnaissance or avoiding problems associated with rough terrain and short time windows for data collection. Because the transmitter antenna parameters are known a priori, analysis of TEM data differs from that of MT, often involving direct inversion of measured field quantities rather than their ratios. Depth of investigation is typically on the order of 1-1.5 km with good conventional equipment. Modest interpretational success has been met with simple "stitched" 1-D profiles-beneficial because of its low computational cost; but in areas where 3-D structure is prominent, TEM data can also be inverted in full 3-D. In areas of strong near-surface heterogeneity, TEM has been successfully used to minimize troublesome distortion effects seen in MT, and hence, best practices are often to collect both MT and TEM data together. Schlumberger soundings are also used due to their flexibility in experiment deployment and the information content of the signal itself (Georgsson 2009).

\section{ELECTRICAL SELF POTENTIAL}

Another electrical method, self-potential (SP), can be used to map boundaries and faults in the early reconnaissance of a geothermal system and has been used to estimate reservoir thickness (useful in reservoir modeling). SP is not widely used due to difficulty in accurate data interpretation (Georgsson 2009).

\section{SURFACE MANIFESTATIONS}

During the early stages of exploration, where no deep wells have been drilled and MT surveys have not yet been conducted, the distribution of thermal features and associated hydrothermal alteration can be used to provide initial constraints on the areal extent of a geothermal system. The distribution of thermal features and major structures are also important components of conceptual models for geothermal systems (Cumming 2009a). Fumaroles are typically located above the upflow zones of geothermal systems whereas chloride hot springs tend to be located in outflow zones that may be laterally displaced from the high-temperature geothermal reservoir. Many thermal manifestations are associated with permeable features such as faults and fracture zones; however, fault zones can also serve as bounding features. Because the extent of a geothermal reservoir is not identifiable at the surface, only crude estimates of volume result from field mapping of the geologic area without additional tests. The use of thermal features to delineate a resource outline is not applicable to hidden geothermal systems, which, by definition, have no visible surface expression.

The addition of remote sensing methods, by virtue of estimating thermal disturbances, provides a more comprehensive estimate than surface manifestations alone-but this is an estimate, not a confirmation, 
of the lateral extent of a heat resource. Remote sensing techniques can also be used to identify zones of surficial hydrothermal alteration. ${ }^{6}$

\section{FLOW TESTING}

Loss zones encountered while drilling within a geothermal reservoir are indicative of permeable fluid entries that help define the reservoir thickness. These zones can also be identified from spinner logs. The top of the reservoir is often identified by the uppermost fluid entry within a well. The total vertical distance between upper and lower fluid entry zones can be used to estimate the reservoir thickness.

\section{MAGNETIC SURVEYS}

Magnetic anomalies, although successful for the mineral exploration industry, are more challenging for the use of geothermal exploration due to the significant magnetic differences among volcanic rock types and (potentially multiple) episodes of hydrothermal alteration. Nevertheless, magnetic surveys have shown distinct signals for high-temperature geothermal fields (e.g., Namafjall and Krafla, Pálmason 1967) and have been widely used in Icelandic low-temperature fields to identify hidden dikes and faults (Gupta 1980). Aerial magnetic surveys sacrifice some data precision, but provide reasonable estimates of deeper structures or the extent of basins; for locations of finer features and faults, higher-resolution on-the-ground surveys are structured either as transects or grids (Georgsson 2009).

\section{SEISMIC PROFILING}

Seismic methods, particularly active reflection measurements, are widely used throughout the oil and gas industry to glean information on the density, porosity, boundaries, and discontinuities of the subsurface. The complexity of geothermal systems in crystalline rocks and volcanic terrains makes it difficult to achieve interpretable information from seismic surveys; however, unsurprisingly, application to geothermal systems in sedimentary formations has been successful, such as the Basin and Range and northeast Iceland (Georgsson 2009; Georgsson and Fridleifsson 2000). Passive seismic methods are more commonly applied to identify the depth to the heat source through measurements of natural seismic activity, which indicates zones of permeability and active faults. Additionally, since S-waves cannot travel through liquids and are shortened in partial melt, the identification of S-wave shadows has been shown to be a consistent method of identifying the depth and location of magma chambers within Iceland's Krafla geothermal field (Árnason et al. 2009; Einarsson 1978). In geothermal systems requiring fluid injection as a means of reservoir stimulation to increase fracture permeability, such as enhanced geothermal systems, passive seismic is a particularly important tool for determining the location of the stimulated reservoir due to the strong direct correlation between reservoir pressure, injection rate, and seismicity (Cloetingh and Negendank 2010).

\section{HYDROTHERMAL ALTERATION MINERAL ASSEMBLAGES}

Examinations of the zonation of hydrothermal alteration in geothermal well core stratigraphy can also provide insights into reservoir volume. Argillic alteration is indicative of the clay cap that develops on top of a geothermal reservoir whereas higher-temperature propylitic alteration (typically characterized

\footnotetext{
${ }^{6}$ Validation of remote-sensing techniques by field observation has shown that a combination of thermal infrared measuring surface temperature anomalies and infrared band ratio analysis measuring reduced vegetation density can provide an $87.5 \%$ accurate location of geothermal manifestations (Atmoko n.d.).
} 
by minerals such as epidote, quartz, chlorite, illite, albite, adularia, wairakite, and calcite) can be diagnostic of a high-temperature geothermal reservoir (Browne 1978). Taken together, these observations can inform a conceptual model of the subsurface used to estimate reservoir volume.

\section{GRAVITY SURVEYS}

Gravity anomalies directly inform an assessment of depth to different formations, and in the process, can identify faults and other structural features. Gravity surveys are an extension of structural mapping, providing an estimate of subsurface geometries through differences in relative gravity between readings of formation densities and the location's surface. In the case of geothermal exploration, gravity anomalies point to areas of hydrothermal alteration and metamorphism, but also, gravity differences may identify the depth to areas of magmatic activity (Gupta 1980; Lyatsky 2010; Hanna et al. 1994).

\section{CORE STRATIGRAPHY}

The above methods rely heavily on the existence of an alteration clay cap (which depends on the rock types present in the system). However, the presence of a clay-rich, low-resistivity feature may not be due to an active geothermal system. Instead, the layer could be the result of a fossil hydrothermal system, or from clay-rich sediments that are unrelated to hydrothermal alteration.

The mineral assemblage that will form depends not only on temperature, but also on the composition of the rock and geothermal fluid and the extent of water-rock interaction. Therefore, resistivity that measures mineral alteration characteristics will reflect the mineral (and therefore temperature) at which the alteration formed-which may or may not be an accurate assessment of current conditions (Karlsdóttir et al. 2012). Altered rock types typical of geothermal reservoirs and seals are more challenging to evaluate in isolation, so the quality of analyses available through core stratigraphy is greater than that of well cuttings (Wohletz and Heiken 1992).

\section{PERMEABILITY}

The ideal geothermal system features permeability throughout the entire reservoir volume, which is capped by a low-permeability seal. Although having a distributed nature is important, it is equally if not more important that the fracture system is oriented in a direction of the stress field that allows features to be open, that fracture systems intersect, and that available fractures are not completely filled by alteration or secondary mineralization. Some mineral phases (such as euhedral quartz and bladed calcite) are often indicators of open-space fractures. Favorable structural settings for permeability that combine the above features differ by tectonic regime (Faulds et al. 2011).

Flow and tracer tests provide the best estimates of permeability because they provide quantitative measurements of fluid transport in a given time period. Permeability measurements conducted on core samples only provide constraints on matrix permeability and cannot capture fracture permeabilityoften the key flow parameter.

All other techniques used to estimate where permeable zones might likely occur do not provide ways to actually quantify permeability. For example, measures of resistivity and other geophysical measures may identify approximate areas that are more permeable than others. Distribution of thermal features indicates the minimal areas of permeability required for those features to reach the surface, but do not indicate potential variations in permeability in the subsurface. 
Because faults and fractures can serve as conduits for geothermal heat and fluid transport, the structural distribution and extent of faulting and fracturing is a primary constraint to the location and depth of hydrothermal activity (Curewitz and Karson 1997). Furthermore, geophysical studies of critically stressed fracture and fault characteristics have provided evidence that these structural mechanics serve an important role in characterizing geothermal fields (Barton et al, 1995; Hickman et al. 1997). Initial field studies have shown promising correlations between known, ongoing geothermal activity and tectonic strain accumulation, indicating that geothermal fluid flow could be reasonably predicted through a structural assessment of regional fault planes in some areas (Blewitt et al. 2002). Careful field and subsurface mapping to assess fault geometry, location, and stress kinematics are therefore critical to the success of exploratory drilling at depth.

\section{VOLUME ESTIMATES IN THE VOLUMETRIC METHOD}

The USGS heat-in-place method uses a volumetric approach to estimate the reservoir thermal energy:

$$
q_{R}=\rho C V\left(T_{R}-T_{0}\right)
$$

where $q_{R}$ is the thermal energy, $\rho C$ is the volumetric specific heat of the reservoir rock, $\mathrm{V}$ is the volume of the reservoir, $T_{R}$ is the characteristic reservoir temperature, and $T_{0}$ is the reference temperature (Muffler and Cataldi, 1978; Williams et al., 2008; Williams, 2014). To determine this volume, estimates of the reservoir areal extent and thickness are needed. Renner et al. note in the 1975 USGS assessment of geothermal systems in the United States that "[t]he lack of reliable data concerning areal extent is a serious constraint in this assessment because many estimates of the subsurface areas... differ by more than three orders of magnitude; in contrast, all other parameters vary by less than one order of magnitude. Therefore, the areal extent is the most critical single parameter in estimating the heat content of a system."

Many geothermal systems contain several distinct reservoirs that are isolated laterally and/or vertically. Therefore, the use of area $\times$ thickness to represent a geothermal reservoir is often an oversimplification of the actual reservoir geometry. The heat-in-place method circumvents the issue that not all of this estimated volume is available for production (i.e., some areas may not be permeable) by modifying the area's recovery factor. Herein lies the majority of observed bias in this method; when compared with actual production, estimated recovery factors show significant upward bias in the initial recovery factor assumptions (Grant 2014). In also assuming plant utilization and efficiency factors, the heat-in-place estimate and the recovery factor are frequently combined to generate an estimate of MWe per USGS's method (Williams et al. 2008). Due to the biases mentioned, comparisons between heat-in-place estimates of capacity and actual production have shown margins of error up to $70 \%$ (Grant 2014). 